\title{
Kinematical Conformal Cosmology: Fundamental Parameters from Astrophysical Observations
}

\author{
Gabriele U. Varieschi \\ Department of Physics, Loyola Marymount University, Los Angeles, CA 90045, USA \\ Correspondence should be addressed to Gabriele U. Varieschi, gvarieschi@lmu.edu \\ Received 9 April 2011; Accepted 10 May 2011 \\ Academic Editors: A. Balbi and S. Ettori
}

Copyright ( $) 2011$ Gabriele U. Varieschi. This is an open access article distributed under the Creative Commons Attribution License, which permits unrestricted use, distribution, and reproduction in any medium, provided the original work is properly cited.

\begin{abstract}
We continue the presentation of an alternative cosmology based on conformal gravity, following our kinematical approach to the subject introduced in a recent paper. In line with the assumptions of our model, which proposes a closed-form expression for the cosmic scale factor $R(t)$, we revise the Hubble and deceleration parameters and introduce modified cosmological distances, analyzing in particular the case of the luminosity distance. Our kinematical conformal cosmology is then able to explain the anomalous acceleration of the Pioneer spacecraft, as due to a local region of gravitational blueshift. From the reported data of the Pioneer anomaly, we also compute the current value of our first fundamental parameter, $\gamma_{0}=1.94 \times 10^{-28} \mathrm{~cm}^{-1}$, in line with the original estimate by P. Mannheim of this quantity. Our second fundamental parameter, $\delta_{0}=3.83 \times 10^{-5}$, interpreted as the current value of a cosmological time variable, is derived from a detailed fitting of type Ia supernovae "gold-silver" data, producing Hubble plots of the same quality of those obtained by standard cosmology, but without requiring any dark matter or dark energy contribution. If further experiments will confirm the presence of an anomalous frequency blueshift in the outer region of the solar system, as described by our model, kinematical conformal cosmology might become a viable alternative to standard cosmological theories.
\end{abstract}

\section{Introduction}

This paper is the second part of a project aimed at introducing an alternative cosmology based on conformal gravity (CG), as originally proposed by Weyl [1-3] and recently revisited by Mannheim and Kazanas [4-6]. In the first paper on the subject [7] (paper I, in the following), we presented the mathematical foundations of our new kinematical approach to conformal cosmology. This was based on a critical reanalysis of fundamental astrophysical observations, starting with the cosmological redshift, and on the fact that modern metrology defines our common units of length and time using nongravitational physics, that is, through emission, propagation, and absorption of electromagnetic waves or similar phenomena.

Since the laws of electromagnetism are notoriously invariant under a conformal transformation, we argued that on a cosmological scale a conformal "stretching" of the spacetime might be present and might yield to an effective change in wavelength or frequency of electromagnetic radiation, equivalent to the observed cosmological redshift (or blueshift, if any). As the origin of this presumed conformal stretching of the metric in the Universe can only be gravitational, we searched its connection with existing theories of gravity that allow this possible conformal symmetry. Our attention was focused on Weyl's conformal gravity, since it is the simplest known conformal generalization of Einstein's general relativity (GR). Weyl's theory is also based on the same principles and assumptions of GR, such as the equivalence principle and other foundational concepts.

The complexity of CG, in particular its fourth-order field equations, as opposed to Einstein's second-order equations, has rendered this theory quite intractable until MannheimKazanas (MK) found the first complete solutions, such as the exterior solution for a static, spherically symmetric source $[4,5]$, and they also showed that it reduces to the classic Schwartzschild solution in the limit of no conformal stretching. In addition, the MK solution is able to interpolate smoothly between the classic Schwartzschild solution and 
the Robertson-Walker (RW) metric, through a series of coordinate transformations based on the conformal structure of the theory.

It was precisely this ability to transform from the conformal MK solution to the standard RW metric, used to describe the cosmological expansion, which convinced us that a universal conformal stretching might be able to mimic the expansion of the Universe. The gravitational origin of this conformal stretching should also lead to the change of observed wavelength frequency of cosmic radiation. The principles of general relativity, which still apply to its conformal extension, naturally propose such a mechanism: the gravitational redshift.

We have shown in our first paper how the original MK potential can support this explanation and how the chain of transformations, from static standard coordinates used in the MK solution to the Robertson Walker coordinates, can lead to a unique expression of the cosmic scale factor $R$. In this way, the conformal symmetry of the universe is "kinematically" broken, and the precise amount of stretching at each space-time point can be determined, once certain parameters of the original MK potential are measured.

In this second paper, we will show how we can determine these fundamental parameters using astrophysical data, such as the luminosities of type Ia supernovae (SNe Ia) and others. In this way, our kinematical conformal cosmology might become a viable alternative model for the description of the Universe, with the advantage of avoiding most of the controversial features of the standard model, such as dark energy, dark matter, and inflationary phases and so forth.

In the next section, we will review our cosmological solutions from paper I, then we will obtain expressions for the Hubble constant and deceleration parameter and also revise the definitions of the standard cosmological distances. In Section 3, we will fit current astrophysical data in order to compute our cosmological parameters and check the consistency of our model. Finally, in Section 4, we will explore the immediate consequences of our model, in terms of the behavior of fundamental constants and other physical quantities.

\section{Kinematical Conformal Cosmology}

2.1. Summary of Results from Paper I. In our first paper [7], we essentially worked with two sets of space-time coordinates. We started with static standard coordinates (SSC) $(r, t, \theta, \phi)$ which are used to express the MK solution for a static, spherically symmetric source, and then we have shown how, far away from massive sources, the MK metric can be transformed into the RW one, by employing a new set of space-time coordinates, denoted in bold type $(\mathbf{r}, \mathbf{t}, \theta, \phi)$, where the angular coordinates are not affected by the transformations. The cosmic scale factor can be introduced as a function of both time coordinates as $\mathbf{R}(\mathbf{t})=R(t) / \sqrt{|k|}$, where $k$ is a cosmological parameter, with dimensions of an inverse square length, originally introduced in the MK solution and whose value we will also determine in this work.
All the space-time coordinates can be turned into dimensionless quantities ( $\mathbf{r}$ is already dimensionless) if we use the following definitions:

$$
\begin{gathered}
\alpha=2 \sqrt{|k|} r, \\
\chi=\sqrt{|k|} c\left(t_{0}-t\right), \\
\zeta=\frac{c\left(\mathbf{t}_{0}-\mathbf{t}\right)}{\mathbf{R}\left(\mathbf{t}_{0}\right)},
\end{gathered}
$$

where we use a look-back time $\left(t_{0}-t\right)$ or $\left(\mathbf{t}_{0}-\mathbf{t}\right)$, since we usually observe radiation emitted in the past at coordinates $(r, t)$ or $(\mathbf{r}, \mathbf{t})$, reaching us at the spatial origin and at our current time $\left(r=0, t_{0}\right)$ or $\left(\mathbf{r}=\mathbf{0}, \mathbf{t}_{0}\right)$.

In our first paper, we used the MK metric as a source of a cosmological gravitational redshift, associated with a redshift parameter $z$ and a cosmic scale factor $R$, using the SSC $r$ coordinate. By considering null geodesics and the other coordinate transformations detailed in [7], we were able to write the cosmic scale factor in any of the variables described above, obtaining the following expressions:

$$
\begin{gathered}
1+z=\frac{R(0)}{R(r)}=\left[1+\delta \alpha-\frac{1}{4}\left(1-\delta^{2}\right) \alpha^{2}\right]^{-1 / 2}, \\
1+z=\frac{R\left(t_{0}\right)}{R(t)}=\cosh \chi-\delta \sinh \chi \\
1+z=\frac{\mathbf{R}(\mathbf{0})}{\mathbf{R}(\mathbf{r})}=\sqrt{1+\mathbf{r}^{2}}-\delta \mathbf{r}, \\
1+z=\frac{\mathbf{R}\left(\mathbf{t}_{0}\right)}{\mathbf{R}(\mathbf{t})} \\
=\left[\cos \left(\sqrt{1-\delta^{2} \zeta}\right)+\frac{\delta}{\sqrt{1-\delta^{2}}} \sin \left(\sqrt{1-\delta^{2}} \zeta\right)\right]^{-1} .
\end{gathered}
$$

To avoid possible misunderstandings, the cosmic scale factor $R$ is considered a function of the time coordinate ( $t$ or the associated $\mathbf{t}$ ) as in standard cosmology, but it is expressed also as a function of the radial coordinate $r$ (or $\mathbf{r}$ ) simply because information from past times is brought to us by light emitted at those radial positions. We used in paper I a gravitational redshift mechanism, based on the static MK potential described in terms of $r$, to explain the cosmological redshift, and therefore we have "improperly" defined the scale factor as a function of radial coordinates, as shown in the previous equation.

The solutions in (2) were obtained for the particular case $\mathbf{k}=k / \sqrt{|k|}=-1$, which was found to be the only one associated with a possible redshift of gravitational origin (the other two cases $\mathbf{k}=0,+1$ did not allow for the observed redshift). The detailed analysis of these solutions can be found again in our paper I. Here, we recall that the solutions in (2) are expressed in terms of another cosmological parameter $\delta$ defined as

$$
\delta \equiv \frac{\gamma}{2 \sqrt{|k|}}
$$


The additional quantity $\gamma$ was also introduced in the original MK solution, and the main objective of this paper is to determine the values of these three cosmological parameters $(\delta, \gamma$, and $k)$ linked together by the previous equation.

Mannheim was able to fit galactic rotation curves without the need of dark matter and to estimate the current value of $\gamma$ as $\gamma_{\text {Mannheim }}=3.06 \times 10^{-30} \mathrm{~cm}^{-1}[8]$. In our first paper we argued that the current value of $\gamma$ is probably close to Mannheim's estimate but needs to be computed from more "local" observations. In addition, $k$ should have a negative value ( since $\mathbf{k}=k / \sqrt{|k|}=-1$ ) while $\delta$ is necessarily limited by $-1<\delta<+1$, so that its current value is probably small and positive (see again paper I for details).

Another hypothesis, introduced in our first paper, is to assume that $\delta$ (as well as $\gamma$ and $k$ ) are probably time-varying quantities, over cosmological ages. In fact, we have proposed that the dimensionless parameter $\delta$ might constitute an effective cosmological time, varying from -1 to +1 , so that (2) represents the evolution of the Universe as seen at our "current time" $\delta=\delta\left(t_{0}\right)$. The most general description is obtained by letting $\delta$ vary in the allowed interval, in all the preceding formulas. If this interpretation is correct, it is possible to write the scale factor directly as a function of the variable $\delta$ and of its current value $\delta\left(t_{0}\right)$ as follows:

$$
1+z=\frac{R\left[\delta\left(t_{0}\right)\right]}{R(\delta)}=\sqrt{\frac{1-\delta^{2}\left(t_{0}\right)}{1-\delta^{2}}},
$$

a simple "semicircular" evolution illustrated in Figure 2 of paper I. The complete connections between all these variables are also discussed in details and summarized in Table 1 of our first paper.

However, at this point, the possible time variation of our cosmological parameters is only a heuristic consideration which needs further investigation. The main parts of this paper (Sections 2 and 3) are devoted to the determination of these parameters regardless of their possible variation over cosmological times. Only in Section 4, we will briefly analyze the consequences of a possible nonconstancy of the parameters.

Therefore, the next step is to check our model against current astrophysical data, in order to establish it as a viable alternative to current cosmology. The cosmological parameters introduced above also need to be evaluated and connected to standard cosmological quantities such as the Hubble constant and the deceleration parameter.

2.2. The Hubble Constant and the Deceleration Parameter. One of the goals of standard cosmology is to determine, both theoretically and experimentally, the Hubble constant and the deceleration parameter which are essential to describe the evolution of the Universe. Since in our model the cosmic scale factor $R$ is determined explicitly by (2), it is not difficult to obtain these important parameters.

We recall that in general the Hubble parameter is defined as $H(t)=\dot{R}(t) / R(t)$ and the deceleration parameter as $q(t)=$ $-\ddot{R}(t) / R(t) H^{2}(t)=-\ddot{R}(t) R(t) / \dot{R}^{2}(t)$, with their currenttime values denoted by $H_{0}$ and $q_{0}$. Standard cosmology measurements of the Hubble constant are usually reported as [9]

$$
H_{0}=100 h \mathrm{~km} \mathrm{~s}^{-1} \mathrm{Mpc}^{-1}=3.24 \times 10^{-18} \mathrm{hs}^{-1},
$$

where $h$ is a number between 0.5 and 1 .

Following the model discussed in paper I and briefly reviewed above, we can write $H(t)$ and $q(t)$ by using our fundamental solutions, and we can also express these quantities in terms of either one of the two time coordinates $t$ or t, introduced previously. As already explained in Section 4.2 of [7], our preference goes to the simpler $t$ coordinate, which makes direct contact with our units of time, but we will also consider the other coordinate $\mathbf{t}$ in the following.

We start by using the SSC time coordinate $t$, which is connected to the dimensionless look-back time $\chi=$ $\sqrt{|k|} c\left(t_{0}-t\right)$ as in (1). In paper I, we have seen in (80)- $(82)$ how to express the first, and second-order time derivatives of $R$ in terms of $\chi$, or directly in terms of the redshift parameter $z$. As a consequence, we can easily write $H(t)$ and $q(t)$ also as a function of $\chi$ or $z$,

$$
\begin{aligned}
H(t) & =\sqrt{|k|} c\left(\frac{\sinh \chi-\delta \cosh \chi}{\cosh \chi-\delta \sinh \chi}\right) \\
& = \pm \sqrt{|k|} c \frac{\sqrt{(1+z)^{2}-\left(1-\delta^{2}\right)}}{(1+z)} \\
q(t) & =\left(\frac{\cosh \chi-\delta \sinh \chi}{\sinh \chi-\delta \cosh \chi}\right)^{2}-2 \\
& =\frac{(1+z)^{2}}{(1+z)^{2}-\left(1-\delta^{2}\right)}-2,
\end{aligned}
$$

where, as in the preceding equations, we use the "current" value $\delta=\delta\left(t_{0}\right)$ (or the value at the time the observations were made). The current-time values of the Hubble and deceleration parameters are obtained in the limit for $\chi \rightarrow 0$ or $z \rightarrow 0$,

$$
\begin{gathered}
H\left(t_{0}\right)=-\frac{\gamma}{2} c, \quad H(z=0)= \pm \frac{\gamma}{2} c, \\
q\left(t_{0}\right)=q(z=0)=\frac{1}{\delta^{2}}-2 .
\end{gathered}
$$

The signs of the quantities in (6) and (7) can be explained with the help of the red-solid curve in Figure 5 of paper I, which represents the ratio $R(\chi) / R\left(\chi_{0}\right)$, or equivalently $R(t) / R\left(t_{0}\right)$, over different cosmological epochs. This bellshaped curve was plotted for a positive value $\delta=\delta\left(t_{0}\right)$ and shows a local blueshift area in the "past" evolution of the Universe, extending back to a time $t_{\mathrm{rs}}$, followed by a redshift region which extends indefinitely to past times and which should represent the observed cosmological redshift from past cosmological epochs.

While we will explain the local blueshift region later in this paper (in Section 3.1), we simply remark here that this red-solid curve in Figure 5 of paper I is symmetric around the point of maximum. Therefore, for each value of $z$, that is, for each value of $R(\chi) / R\left(\chi_{0}\right)$, we have two corresponding 
values of the Hubble parameter (except at the maximum, for $z_{\min }=\sqrt{1-\delta^{2}}-1$, where $H=0$ ), and the two related points on the curve, at the same redshift level, will have equal and opposite expansion rates. This yields the double sign in the previous expressions for $H$, when given as a function of $z$.

This argument applies also to the $z=0$ case, corresponding to the current time $t_{0}$ at which $H\left(t_{0}\right)=-(\gamma / 2) c$ is negative, but also referring to a time in the past $\left(t_{\mathrm{rs}}\right)$ at which we start observing the cosmological redshift, with $H\left(t_{\mathrm{rs}}\right)=$ $+(\gamma / 2) c$, a positive quantity. This does not contradict the current estimates of $H_{0}$ as a positive quantity. They are based on redshift observations of light coming from galaxies at times in the past $t \lesssim t_{\mathrm{rs}}$; therefore, what we call $H_{0}$ in standard cosmology should be actually indicated as $H\left(t_{\mathrm{rs}}\right)=$ $+(\gamma / 2) c$, again a positive quantity.

In Section 3.1, we will evaluate the current value of the gamma parameter, by analyzing the local blueshift in the region of our solar system, corresponding to a negative $H\left(t_{0}\right)=-(\gamma / 2) c$. In this way, we will be able to estimate the gamma parameter as $\gamma\left(t_{0}\right) \cong 1.94 \times 10^{-28} \mathrm{~cm}^{-1}$, and this will allow us to evaluate also the Hubble parameter at the beginning of the redshift region (time $t_{\mathrm{rs}}$ ), by using the same value of $\gamma$ and the positive sign in (7), or equivalently assuming by symmetry $\gamma\left(t_{\mathrm{rs}}\right) \cong-1.94 \times 10^{-28} \mathrm{~cm}^{-1}$ and using $H\left(t_{\mathrm{rs}}\right)=-\left(\gamma\left(t_{\mathrm{rs}}\right) / 2\right) c$. Numerically, we estimate

$$
\begin{gathered}
H\left(t_{0}\right)=-\frac{\gamma\left(t_{0}\right)}{2} c \cong-2.91 \times 10^{-18} \mathrm{~s}^{-1}, \\
H\left(t_{\mathrm{rs}}\right)=+\frac{\gamma\left(t_{0}\right)}{2} c=-\frac{\gamma\left(t_{\mathrm{rs}}\right)}{2} c \cong+2.91 \times 10^{-18} \mathrm{~s}^{-1},
\end{gathered}
$$

where again the details of this analysis will be presented later in this paper.

The connection with the standard cosmology value of $H_{0}$ in (5) is immediate; all the standard astrophysical observations which led to the existing estimates of $H_{0}$ were done by observing celestial objects in the redshift region, therefore for $t \lesssim t_{\text {rs }}$ or $r \gtrsim r_{\text {rs }}$, where $r_{\text {rs }}$ is the distance at which we start observing the cosmological redshift. Therefore,

$$
\begin{gathered}
H_{0}=H\left(t_{\mathrm{rs}}\right)=+\frac{\gamma\left(t_{0}\right)}{2} c \cong+2.91 \times 10^{-18} \mathrm{~s}^{-1} \\
=100 h_{\mathrm{rs}} \mathrm{km} \mathrm{s}^{-1} \mathrm{Mpc}^{-1}, \\
h_{\mathrm{rs}} \cong 0.897,
\end{gathered}
$$

in line with the current estimates of $H_{0}$ [9]. Our value, $h_{\mathrm{rs}} \cong 0.897$, is the direct estimate of the $h$ parameter based on the Pioneer anomaly data (see Section 3.1) and is close to recent determinations by the Wilkinson Microwave Anisotropy Probe (WMAP) $[10] h_{\mathrm{WMAP}} \cong 0.73 \pm 0.03$, by the Hubble Space Telescope Key Project (HST Key Project) [11] $h_{\mathrm{HST}} \cong 0.72 \pm 0.08$, and others [12]

The deceleration parameter at current time $t_{0}$ or at time $t_{\mathrm{rs}}$ (in both cases $z=0$ ) is given by (7) as a function of the dimensionless $\delta$, which cannot be estimated from the Pioneer data. We cannot use results for $q$ or similar acceleration parameters coming from standard cosmology, such as those obtained from type Ia supernovae analysis, as they are based on totally different assumptions. We will have to analyze and reinterpret the concepts of standard candle, luminosity distance, and so forth, before we can estimate $\delta\left(t_{0}\right)$ and therefore $q\left(t_{0}\right)$. This will be done in the following sections.

Before we proceed in this direction, we also study the expressions for the Hubble and deceleration parameters which can be obtained by using other variables. For example, we can recalculate our expressions using the RW time variable $\mathbf{t}$, that is, define the Hubble parameter as $\mathbf{H}(\mathbf{t})=\dot{\mathbf{R}}(\mathbf{t}) / \mathbf{R}(\mathbf{t})$ and the deceleration parameter, $\mathbf{q}(\mathbf{t})=$ $-\ddot{\mathbf{R}}(\mathbf{t}) / \mathbf{R}(\mathbf{t}) \mathbf{H}^{2}(\mathbf{t})=-\ddot{\mathbf{R}}(\mathbf{t}) \mathbf{R}(\mathbf{t}) / \dot{\mathbf{R}}^{2}(\mathbf{t})$, where the boldface symbols denote quantities related to the RW metric, as discussed in the previous section and in paper I. It is straightforward to obtain expressions similar to those in (6),

$$
\begin{aligned}
\mathbf{H}(\mathbf{t})= & -\frac{c \sqrt{1-\delta^{2}}}{\mathbf{R}\left(\mathbf{t}_{0}\right)} \\
& \times\left[\frac{\delta \cos \left(\sqrt{1-\delta^{2}} \zeta\right)-\sqrt{1-\delta^{2}} \sin \left(\sqrt{1-\delta^{2}} \zeta\right)}{\sqrt{1-\delta^{2}} \cos \left(\sqrt{1-\delta^{2}} \zeta\right)+\delta \sin \left(\sqrt{1-\delta^{2}} \zeta\right)}\right] \\
= & \pm \frac{c}{\mathbf{R}\left(\mathbf{t}_{0}\right)} \sqrt{(1+z)^{2}-\left(1-\delta^{2}\right)}, \\
\mathbf{q}(\mathbf{t})= & {\left[\frac{\sqrt{1-\delta^{2}} \cos \left(\sqrt{1-\delta^{2}} \zeta\right)+\delta \sin \left(\sqrt{1-\delta^{2}} \zeta\right)}{\delta \cos \left(\sqrt{1-\delta^{2}} \zeta\right)-\sqrt{1-\delta^{2}} \sin \left(\sqrt{1-\delta^{2}} \zeta\right)}\right]^{2} } \\
= & \frac{\left(1-\delta^{2}\right)}{(1+z)^{2}-\left(1-\delta^{2}\right)},
\end{aligned}
$$

where again $\delta=\delta\left(t_{0}\right)$. We use here the dimensionless variable $\zeta=c\left(\mathbf{t}_{0}-\mathbf{t}\right) / \mathbf{R}\left(\mathbf{t}_{0}\right)$, or we express the Hubble and deceleration parameters directly in terms of $z$.

The current-time values of these two parameters are obtained in the limit for $\zeta \rightarrow 0$ or $z \rightarrow 0$, respectively,

$$
\begin{gathered}
\mathbf{H}\left(\mathbf{t}_{0}\right)=-\frac{c}{\mathbf{R}\left(\mathbf{t}_{0}\right)} \delta, \quad \mathbf{H}(z=0)= \pm \frac{c}{\mathbf{R}\left(\mathbf{t}_{0}\right)} \delta, \\
\mathbf{q}\left(\mathbf{t}_{0}\right)=\mathbf{q}(z=0)=\frac{1}{\delta^{2}}-1,
\end{gathered}
$$

with the same interpretation which was given for (7). These expressions for the Hubble and deceleration parameters in the two temporal variables are obviously connected. It follows from the definitions that $\mathbf{H}(\mathbf{t})=H(t)(d t / d \mathbf{t})$ and $\mathbf{q}(\mathbf{t})=$ $q(t)-\left(d^{2} t / d \mathbf{t}^{2}\right) / H(t)(d t / d \mathbf{t})^{2}$, with the derivatives between time variables given by

$$
\begin{aligned}
\frac{d t}{d \mathbf{t}}= & \frac{\sqrt{1-\delta^{2}}}{\sqrt{|k|} \mathbf{R}\left(\mathbf{t}_{0}\right)} \\
& \times \frac{1}{\left[\sqrt{1-\delta^{2}} \cos \left(\sqrt{1-\delta^{2}} \zeta\right)+\delta \sin \left(\sqrt{1-\delta^{2}} \zeta\right)\right]} \\
= & \frac{(1+z)}{\sqrt{|k|} \mathbf{R}\left(\mathbf{t}_{0}\right)},
\end{aligned}
$$




$$
\begin{aligned}
\frac{d^{2} t}{d \mathbf{t}^{2}}= & \frac{c\left(1-\delta^{2}\right)}{\sqrt{|k| \mathbf{R}^{2}\left(\mathbf{t}_{0}\right)}} \\
& \times \frac{\left[\delta \cos \left(\sqrt{1-\delta^{2}} \zeta\right)-\sqrt{1-\delta^{2}} \sin \left(\sqrt{1-\delta^{2}} \zeta\right)\right]}{\left[\sqrt{1-\delta^{2}} \cos \left(\sqrt{1-\delta^{2}} \zeta\right)+\delta \sin \left(\sqrt{1-\delta^{2}} \zeta\right)\right]^{2}} \\
= & \mp \frac{c(1+z)}{\sqrt{|k|} \mathbf{R}^{2}\left(\mathbf{t}_{0}\right)} \sqrt{(1+z)^{2}-\left(1-\delta^{2}\right)},
\end{aligned}
$$

so that the connecting formulas can be easily derived.

Similarly, we could also write Hubble and deceleration parameters in terms of space variables $r, \alpha$, or $\mathbf{r}$, since we have in (2) the cosmic scale factor $R$ expressed in all these variables, but these expressions would not be very useful because the experimental values of $H_{0}$ and $q_{0}$ are usually referred only to the temporal variables. We will return in Section 3.1 on the connection with experimental observations.

Finally, from (4), it is possible to introduce $H$ and $q$ directly as a function of our cosmological time $\delta$,

$$
\begin{gathered}
H(\delta)=\frac{1}{R(\delta)} \frac{d R}{d \delta}=-\frac{\delta}{1-\delta^{2}}, \\
q(\delta)=-\frac{\left(d^{2} R / d \delta^{2}\right) R(\delta)}{(d R / d \delta)^{2}}=\frac{1}{\delta^{2}},
\end{gathered}
$$

and the expressions in (6) and (10) will reduce to those in (13), using the transformations outlined in Table 1 of paper I.

\subsection{Luminosity Distance and Other Cosmological Distances.}

Before we make contact with experimental data, especially with standard candle measurements, we need to review the definitions of the cosmological distances, following the new principles of our kinematical conformal cosmology as outlined above and in paper I.

Several distances are usually introduced in standard cosmology and used in conjunction with astronomical observations in order to establish the "cosmological distance ladder," that is, the different steps employed to measure astronomical distances and the size of the Universe (for an extensive introduction to the subject, see $[13,14])$. This process started historically at the time of Greek astronomy with the determination of the size and radius of our planet and with the first approximate measurements of the astronomical unit and other distances within the solar system.

These estimates, based mainly on parallax methods, were later refined by modern astronomers and extended to parallax measurements of nearby stars. These geometrical methods led to the introduction of distances such as the parallax distance $d_{P}$, whose definition is not affected by our alternative approach to cosmology. As defined in Weinberg's books $[15,16]$,

$$
d_{P} \equiv \frac{b}{\theta}=\mathbf{R}\left(\mathbf{t}_{0}\right) \frac{\mathbf{r}}{\sqrt{1-\mathbf{k r}^{2}}}=\mathbf{R}\left(\mathbf{t}_{0}\right) \frac{\mathbf{r}}{\sqrt{1+\mathbf{r}^{2}}},
$$

where $b$ is the impact parameter of light reaching the observer from a (parallax) distance $d_{P}$ with parallax angle $\theta$, and we have used our preferred value $\mathbf{k}=-1$ in the righthand side of the last equation (see $[9,14,15]$ or [16], for full details on all these distances).

Two more fundamental distances are usually introduced in cosmology, the comoving distance $d_{C}$ (sometimes also called coordinate or effective distance) and the proper distance $d_{\text {prop }}$ :

$$
\begin{gathered}
d_{C} \equiv \mathbf{R}\left(\mathbf{t}_{0}\right) \mathbf{r}, \\
d_{\text {prop }} \equiv \mathbf{R}(\mathbf{t}) \int_{0}^{\mathbf{r}} \frac{d \mathbf{r}^{\prime}}{\sqrt{1-\mathbf{k r}^{\prime 2}}}=\mathbf{R}(\mathbf{t}) \chi \\
=\mathbf{R}(\mathbf{t})\left\{\begin{array}{cc}
\arcsin \mathbf{r} ; & \mathbf{k}=+1 \\
\mathbf{r} ; & \mathbf{k}=0 \\
\operatorname{arcsinh} \mathbf{r} ; & \mathbf{k}=-1,
\end{array}\right\},
\end{gathered}
$$

where $d_{C}$ usually refers to the current-time expansion factor $\mathbf{R}\left(\mathbf{t}_{0}\right)$, while $d_{\text {prop }}$ can refer to any cosmological time $\mathbf{t}$ being considered. These definitions are also unchanged in our cosmology and can be rewritten in terms of other variables using the transformations in paper I.

Continuing our brief summary of the cosmological distance ladder, modern parallax techniques can determine star distances up to about 100 parsec $\left(1 \mathrm{pc}=3.086 \times 10^{18} \mathrm{~cm}\right)$, and these distance estimates are usually combined with the measured apparent star luminosities, to obtain their corresponding absolute luminosities. For this purpose, the inverse square law is typically assumed, introducing the luminosity distance $d_{L}$ and connecting it to the apparent $(l)$ and absolute bolometric luminosity $(L)$ of a light source as follows:

$$
\begin{gathered}
l=\frac{L}{4 \pi d_{L}^{2}}, \\
d_{L} \equiv \sqrt{\frac{L}{4 \pi l}}=\frac{\mathbf{R}^{2}\left(\mathbf{t}_{0}\right)}{\mathbf{R}(\mathbf{t})} \mathbf{r}=(1+z) \mathbf{R}\left(\mathbf{t}_{0}\right) \mathbf{r} .
\end{gathered}
$$

This definition follows from the consideration that "In a Euclidean space the apparent luminosity of a source at rest at distance $d$ would be $L / 4 \pi d^{2}, \ldots$ " (see again Weinberg [15, Section 14.4]). In the second line of the previous equation, the factor $\mathbf{R}\left(\mathbf{t}_{0}\right) / \mathbf{R}(\mathbf{t})=(1+z)$ appears due to the standard redshift interpretation, namely, that photons of energy $h \nu$ are redshifted to energy $h \nu \mathbf{R}(\mathbf{t}) / \mathbf{R}\left(\mathbf{t}_{0}\right)$ and that the time interval of photon emission $\delta t$ is also changed to $\delta t \mathbf{R}\left(\mathbf{t}_{0}\right) / \mathbf{R}(\mathbf{t})$. The total power emitted (energy per unit time) is therefore redshifted by a combined factor $\mathbf{R}^{2}(\mathbf{t}) / \mathbf{R}^{2}\left(\mathbf{t}_{0}\right)=(1+z)^{-2}$ which will enter the denominator of the square root of (16), thus resulting in the final $(1+z)$ factor in the equation (see [15] for full details). In other words, the standard redshift effect is assumed to alter the apparent luminosity of a standard candle placed far away, so that the $(1+z)$ factor corrects for this effect, while the absolute luminosity $L$ of our candle is considered fixed and constant. We will need to change this view in our alternative interpretation.

Before we consider our revised luminosity distance, we recall that astronomical luminosity measurements are usually expressed in terms of magnitudes $(m)$ using Pogson's 
law, $m_{1}-m_{2}=-2.5 \log _{10}\left(l_{1} / l_{2}\right)$, for any two apparent luminosities. Traditionally, the absolute bolometric luminosity $L$ of a standard candle is defined as the apparent bolometric luminosity of the same object placed at a reference distance of $10 \mathrm{pc}$, so that the distance modulus $\mu$ (difference between the apparent and absolute bolometric magnitudes) will result as follows:

$$
\mu \equiv m-M=5 \log _{10}\left(\frac{d_{L}}{\mathrm{pc}}\right)-5=5 \log _{10}\left(\frac{d_{L}}{\mathrm{Mpc}}\right)+25 .
$$

In standard cosmology, the luminosity distance on the right-hand side of the last equation is then expressed as a function of $z$ and other cosmological parameters, such as the density parameters $\Omega_{M}$ and $\Omega_{\Lambda}$, that is, $d_{L}=d_{L}\left(z ; \Omega_{M}, \Omega_{\Lambda}\right)$. The comparison with experimental observations is usually carried out by fitting the luminosity distance expression to the measured distance moduli $(\mu)$ for several astrophysical light sources, which can be considered standard candles, that is, assumed having constant intrinsic luminosity. This method has proven to be particularly reliable when applied to type Ia supernovae and will be analyzed in detail in the following sections.

However, our view of the luminosity distance is different from the one outlined above. First of all, the cosmological redshift is related to the intrinsic stretching of the space-time fabric at cosmological distances and over cosmological times. This is realized through the gravitational redshift mechanism described in (2) and not anymore through a Dopplerlike shift in photons energy or change in their emission frequency. In this view, the correcting factor $(1+z)$ inserted in (16) and described above is no longer necessary; the photons are emitted at the source with the precise frequency, energy, and rate of emission measured by the Earth's observer (although these differ from the same quantities measured by an observer near the source).

Therefore, we correct the standard definition of the luminosity distance in $(16)$ by removing the $(1+z)$ factor

$$
\begin{aligned}
d_{L} & \equiv \sqrt{\frac{L_{z}}{4 \pi l}}=\mathbf{R}\left(\mathbf{t}_{0}\right) \mathbf{r} \\
& =\mathbf{R}\left(\mathbf{t}_{0}\right) \frac{\delta(1+z)+\sqrt{(1+z)^{2}-\left(1-\delta^{2}\right)}}{\left(1-\delta^{2}\right)}
\end{aligned}
$$

where we have inserted, on the right-hand side of the equation, our expression for the coordinate $\mathbf{r}$, introduced in (76) of our paper I, as a function of our current value $\delta=\delta\left(t_{0}\right)$. We also choose the positive sign in front of the square root to select the solution corresponding to past redshift, $z>0$ for $\mathbf{r}>\mathbf{r}_{\mathrm{rs}}=2 \delta /\left(1-\delta^{2}\right)$, which is the correct choice for the subsequent analysis (see [7] for details).

In the previous equation, we have also indicated a dependence on $z$ of the absolute luminosity $L_{z}$ of the standard candle being considered (indicated by the subscript $z$ ). This is the second fundamental difference in our analysis of the luminosity distance. We have based our discussion in paper I on the hypothesis that the fundamental units of measure, such as the meter, the second, or others, depend on the space-time position in the Universe and differ from the same units of measure at another space-time location. Consequently, we have to assume that the same hypothesis applies to the luminosity of a "standard candle," that is, we cannot assume anymore that $L$ is an invariant quantity, when observing these candles placed at cosmological distances.

In our first paper, we also postulated that space or time intervals are "dilated" by the $(1+z)$ factor, when referring to a cosmological location characterized by redshift $z$, but we did not introduce any similar dependence for the third fundamental mechanical quantity, that is, mass. In fact, we have no a priori indication of how masses should scale due to our space-time stretching; therefore, we summarize the scaling properties of the three fundamental quantities as follows:

$$
\begin{gathered}
\delta l_{z}=(1+z) \delta l_{0}, \\
\delta t_{z}=(1+z) \delta t_{0}, \\
\delta m_{z}=f(1+z) \delta m_{0} .
\end{gathered}
$$

The first two lines in this equation simply rewrite (37) of our first paper in a simplified notation. Quantities with the zero subscript represent units or intervals (of space, time, or mass) as measured by an observer at his/her location and time (typically at the origin of space and at time $t_{0}$ as usual). Quantities with the $z$ subscript represent the same units or intervals as "seen" by the observer located at the origin, but when these "objects" are placed at a cosmological location characterized by redshift $z$. (We have avoided so far this subscript notation (also carefully avoided in paper I) because it is easily confused with the standard cosmology notation, where the zero subscript normally indicates the observed (redshifted) quantity, as opposed to the nonredshifted quantity (usually with no subscript). On the contrary, in our new notation, the "redshifted" quantity $q$ acquires a $z$ subscript $\left(q_{z} \equiv q(t)\right.$, observed at the origin, but with information coming from past time $t$ ), while the "nonredshifted" quantity acquires the zero subscript $\left(q_{0} \equiv\right.$ $q\left(t_{0}\right)$, observed at the origin, at current time $\left.t_{0}\right)$. Since this subscript notation is much more compact than our previous one, we will adopt it for the rest of this paper. For instance, we will write the current values of our cosmological parameters as $\delta_{0} \equiv \delta\left(t_{0}\right), \gamma_{0} \equiv \gamma\left(t_{0}\right)$, etc.) Space-time intervals are dilated by the $(1+z)$ factor, simply due to our new interpretation of the cosmological redshift.

In the equation for mass (third line of (19)), we have left the dependence on $z$ totally undetermined, assuming that some function $f(1+z)$ will connect units of mass at different locations in the Universe. We only suppose that $f$ will be a function of the redshift factor $(1+z)$, such that for $z=0$, it reduces to unity, that is, $f(1)=1$, leaving masses unchanged at locations where $z=0$. We will determine this function $f$ in the following.

The scaling of the fundamental mechanical quantities in (19) will be reflected in similar properties of any other mechanical quantity or unit. We consider, for example, the case of energy, as the luminosity discussed above is just 
energy emitted per unit time. Since $1 \mathrm{erg}=1 \mathrm{~g} \mathrm{~cm}^{2} \mathrm{~s}^{-2}$, an amount of energy can be written as $\delta E \sim \delta m \delta l^{2} \delta t^{-2}$, and it is immediate to check that energies will scale like masses, following the last equation. We can therefore write

$$
\delta E_{z}=f(1+z) \delta E_{0},
$$

which implies that the total energy emitted by a standard candle placed at redshift $z$ would be measured by the observer at the origin to be different from the total energy emitted by the same candle when placed at the origin. Since these energies are emitted during some (finite) intervals of time, we can write $\delta E_{z}=\int L_{z}\left(t_{z}\right) d t_{z}=f(1+z) \delta E_{0}=$ $f(1+z) \int L_{0}\left(t_{0}\right) d t_{0}$, where the luminosities are also labeled like all the other quantities and refer to times connected by the same dilation factor $t_{z}=t_{0}(1+z)$. (In the standard interpretation, $t_{0}$ would be considered as the time $t_{\text {rest }}$ in the candle's restframe of reference and $t_{z}$ as the time $t_{\mathrm{obs}}$ measured by the observer who sees the candle "moving" due to the expansion of the Universe. Standard relativistic time dilation would yield $t_{\mathrm{obs}}=t_{\mathrm{rest}}(1+z)$. This time dilation effect, which has been observed in the evolution of type Ia $\mathrm{SNe}$ [17-20], is also present in our theory although its interpretation is the one given by our fundamental (19).) Since the infinitesimal time intervals are similarly related, $d t_{z}=d t_{0}(1+z)$, the two luminosities are connected in the following way:

$$
L_{z}\left(t_{z}\right)=\frac{f(1+z)}{(1+z)} L_{0}\left(t_{0}\right) .
$$

Our new definition of luminosity distance will therefore generalize the original definition in (16), by using the actual luminosity $L_{z}$, instead of $L_{0}$, to correct for the intrinsic changes in the candle's energy output. To obtain the full expression of this distance, we use (18) and (21) together

$$
\begin{aligned}
d_{L} & =\sqrt{\frac{L_{z}}{4 \pi l}}=\sqrt{\frac{f(1+z)}{(1+z)} \frac{L_{0}}{4 \pi l}}=\mathbf{R}_{0} \mathbf{r} \\
& =\mathbf{R}_{0} \frac{\delta_{0}(1+z)+\sqrt{(1+z)^{2}-\left(1-\delta_{0}^{2}\right)}}{\left(1-\delta_{0}^{2}\right)},
\end{aligned}
$$

where we also used our simplified notation for the parameters $\delta_{0} \equiv \delta\left(t_{0}\right), \mathbf{R}_{0} \equiv \mathbf{R}\left(\mathbf{t}_{0}\right)$, as previously discussed.

Comparing our new expression with the original one in (16), we note that basically we replaced the $(1+z)$ factor with a more complex factor $\sqrt{(1+z) / f(1+z)}$, where again the function $f(1+z)$ is still undetermined at this point. This is because the standard definition would use the "constant" luminosity $L_{0}$, as $d_{L}=\sqrt{L_{0} / 4 \pi l}=\sqrt{(1+z) / f(1+z)} \mathbf{R}_{0} \mathbf{r}$, instead of the "variable" $L_{z}$. One could argue that if the $f$ function was to be equal to $(1+z)^{-1}$, that is, if masses were to scale like $(1+z)^{-1}$, we would recover the original definition, but this is not exactly the case. Again, the original definition (16) assumes an invariant value $L=L_{0}$ of the standard candle's luminosity at all cosmological locations, while our new definition (22) is based on the choice of $L_{z}$ as the standard candle's luminosity, and in general this quantity is not invariant anymore but changes according to (21).
In other words, we could have defined instead

$$
\begin{aligned}
d_{L} & =\sqrt{\frac{L_{0}}{4 \pi l}}=\sqrt{\frac{(1+z)}{f(1+z)}} \mathbf{R}_{0} \mathbf{r} \\
& =\sqrt{\frac{(1+z)}{f(1+z)}} \mathbf{R}_{0} \frac{\delta_{0}(1+z)+\sqrt{(1+z)^{2}-\left(1-\delta_{0}^{2}\right)}}{\left(1-\delta_{0}^{2}\right)},
\end{aligned}
$$

using $L_{0}$ instead of $L_{z}$, and then supplementing this definition with the information of (21). We will see that this will not affect the subsequent discussion on type Ia supernovae. In any case, we prefer our definition in (22) as it corrects the distance estimates, due to the variability of the candle's luminosity. For example, if a candle is intrinsically dimmed when placed far away, its distance should be smaller than the one estimated with the original definition, given the same value $l$ for the observed apparent luminosity. Our new definition (22) precisely incorporates such corrections and will imply a revision of the current distance estimates based on luminosity measurements.

It will be useful in the following to consider also the spectral energy distribution, $F(\lambda) \equiv d L / d \lambda$. Following (21), we can write $\int F_{z}\left(\lambda_{z}, t_{z}\right) d \lambda_{z}=(f(1+z) /(1+z)) \int F_{0}\left(\lambda_{0}, t_{0}\right) d \lambda_{0}$, where the meaning of the subscripts is the same as in the preceding equations. The wavelength variables and related differentials are connected as usual, $\lambda_{z}=\lambda_{0}(1+z)$ and $d \lambda_{z}=d \lambda_{0}(1+z)$, so that the direct relation between $F_{z}$ and $F_{0}$ is

$$
F_{z}\left(\lambda_{z}, t_{z}\right)=\frac{f(1+z)}{(1+z)^{2}} F_{0}\left(\lambda_{0}, t_{0}\right) .
$$

After discussing at length the modifications to the luminosity distance, we return for completeness to the other definitions of cosmological distances. Two other distances are usually introduced, the angular diameter distance $d_{A}$, when an extended light source of true proper diameter $D$ is placed at a (angular) distance $d_{A}$ and observed having an angular diameter $\vartheta$, and the proper-motion distance $d_{M}$, when proper motions in the direction transverse to the line of sight are considered. Their standard definitions are [15]

$$
\begin{gathered}
d_{A} \equiv \frac{D}{\vartheta}=\mathbf{R}(\mathbf{t}) \mathbf{r}=\frac{\mathbf{R}_{0} \mathbf{r}}{(1+z)}, \\
d_{M} \equiv \frac{V_{\perp}}{\Delta \vartheta / \Delta t_{\mathrm{obs}}}=\mathbf{R}_{0} \mathbf{r},
\end{gathered}
$$

where, in the second definition, $V_{\perp}$ is the true velocity of the source in the direction perpendicular to the line of sight, and $\Delta \vartheta$ is the change in the (angular) position of the object during the time interval of observation $\Delta t_{\mathrm{obs}}$.

Both definitions need to be reconsidered in our new interpretation. In the first definition, the "true" diameter of the light source will depend on $z$ as for all the other lengths, $D_{z}=(1+z) D_{0}$, but using the RW metric, the proper distance across the source is $D_{z}=\mathbf{R}(\mathbf{t}) \mathbf{r} \vartheta$ for small angular diameters $\vartheta \ll 1$, which leads essentially to the same expression as in the standard treatment, just with $D$ replaced by $D_{z}$. Therefore, our definition of $d_{A}$ is similar to the standard one, 
but needs to be supplemented by the scaling equation of the source diameter

$$
\begin{aligned}
d_{A} \equiv \frac{D_{z}}{\vartheta} & =\mathbf{R}(\mathbf{t}) \mathbf{r}=\frac{\mathbf{R}_{0} \mathbf{r}}{(1+z)}, \\
D_{z} & =(1+z) D_{0} .
\end{aligned}
$$

In this way, the right-hand side of the equation connecting $d_{A}$ to the cosmological quantities is unaffected, but when we make contact with observations, that is, we use the left-hand side of the equation, the change of the true diameter with $z$ must be included. Since $D_{z}$ increases with (positive) $z$, using the old definition, which assumes a fixed $D=D_{0}$, would result in underestimating the diameter distances by a factor $(1+z)$.

Similar care has to be taken in revising the proper motion distance $d_{M}$. A moving source will have a transverse velocity $V_{\perp}$ which is unaffected by our (19), since it is a ratio between quantities which scale in the same way. In the standard theory, the time interval of observation $\Delta t_{\mathrm{obs}}$ is thought to be different from the time interval of motion $\Delta t_{\text {mot }}$ due to the usual redshift factor, $\Delta t_{\mathrm{mot}}=\Delta t_{\mathrm{obs}} /(1+z)$. However, in our view, the observed time $\Delta t_{\mathrm{obs}}$ is precisely the true time interval during which the object moved at a redshift $z$ : $\Delta t_{\mathrm{obs}}=\Delta t_{z}$. The proper distance travelled is $\Delta D_{z}=V_{\perp} \Delta t_{z}=$ $V_{\perp} \Delta t_{\mathrm{obs}}=\mathbf{R}(\mathbf{t}) \mathbf{r} \Delta \mathcal{V}$, using the RW metric as in the treatment of the angular distance above, and our revised expression of the proper motion distance is

$$
d_{M} \equiv \frac{V_{\perp}}{\Delta \vartheta / \Delta t_{\mathrm{obs}}}=\mathbf{R}(\mathbf{t}) \mathbf{r}=\frac{\mathbf{R}_{0} \mathbf{r}}{(1+z)} .
$$

In this case, we had to modify the right-hand side of the equation (compared to the standard definition), but the lefthand side is unaffected (in particular, $\Delta t_{\mathrm{obs}}=\Delta t_{z}$ is still simply the observation time interval). Finally, we note that our corrected expressions for $d_{A}$ and $d_{M}$ are essentially the same (both lead to the expression $\mathbf{R}(\mathbf{t}) \mathbf{r}$ ) since the geometry is completely equivalent $\left(D_{z}\right.$ is equivalent to $V_{\perp} \Delta t_{\mathrm{obs}}, \vartheta$ to $\Delta \vartheta)$.

Equations (14), (15), (22), (26), and (27) are our revised expressions for the classic distances used in cosmology. As in the case of their standard counterparts, they are all approximately equal to each other for $z \ll 1$ and $\mathbf{r} \ll 1$, that is, $d_{C} \simeq d_{\text {prop }}\left(\mathbf{t}_{0}\right) \simeq d_{P} \simeq d_{L} \simeq d_{A} \simeq d_{M} \simeq \mathbf{R}_{0} \mathbf{r}$. We also note that three of the six definitions of distance were modified by our new kinematical conformal cosmology, thus potentially affecting current astronomical distance estimates.

One final consideration is needed regarding the SSC space coordinate $r$. This dimensionful coordinate can be used to measure distances in the static standard coordinates, which are in general different from the cosmological distances described above. However, for small distances, or for $z \ll 1$, this coordinate $r$ should also reduce to $\mathbf{R}_{0} \mathbf{r}$ as for all the other distances. We recall from paper I that the coordinate transformation between $r$ and $\mathbf{r}$ is

$$
r=\frac{1}{\sqrt{|k|}} \frac{\mathbf{r}}{\sqrt{1+\mathbf{r}^{2}}-\delta_{0} \mathbf{r}} \longrightarrow \frac{\mathbf{r}}{\sqrt{|k|}} \simeq \mathbf{R}\left(\mathbf{t}_{0}\right) \mathbf{r}
$$

where the limit in the last expression is for $\mathbf{r} \ll \mathbf{0}$ and $\delta_{0} \simeq 0$. The previous equation implies that the current value of the scale factor is simply related to the parameter $k$ (if the current value of $\delta_{0}$ is close to zero),

$$
\mathbf{R}_{0}=\mathbf{R}\left(\mathbf{t}_{0}\right) \simeq \frac{1}{\sqrt{\left|k_{0}\right|}},
$$

where, from now on, we will also denote with a zero subscript $\left(k_{0}\right)$ the current value of the $k$ parameter. Equation (27) is particularly important to simplify the connection between the Hubble constants $\mathbf{H}$ and $H$, in view of (6)-(12). It is easy to check that, using the previous equation, the general connection simplifies to

$$
\mathbf{H}(\mathbf{t}) \simeq H(t)(1+z),
$$

so that for $z=0$ the two Hubble constants basically coincide, that is, $\mathbf{H}_{0} \simeq H_{0}$. Again, these results are valid only for $\delta_{0} \simeq 0$, but we will show in the next section that this is actually the case of interest.

It is beyond the scope of this paper to attempt a full revision of the "cosmological distance ladder," in view of our changes in the distance definitions. While we leave this analysis to future work on the subject, we will concentrate our efforts in the next section on applying our revised definition of the luminosity distance to type Ia supernovae.

\section{Cosmological Parameters}

The central part of our analysis will deal with the evaluation of the cosmological parameters in our model: the dimensionless $\delta$ parameter, the $\gamma$ and $k$ parameters (or the original $\kappa$ quantity, see paper I [7]) all measured with reference to the current time $t_{0}$ if these quantities are considered timevarying parameters. (We have already remarked in Section 2.1 that the determination of these parameters is independent of their possible time variation, that is, the results in the following subsections (Sections 3.1, 3.2, and 3.3) are valid also in the case of no time variation of the parameters. In this case, $\delta, \gamma$, and $k$ should be considered simply as universal constants.)

We have already introduced $\gamma_{0}=\gamma\left(t_{0}\right) \cong 1.94 \times$ $10^{-28} \mathrm{~cm}^{-1}$ in Section 2.2, but we still have to show how this value was obtained and compare it to the original evaluation of $\gamma$ done by Mannheim. We will proceed first to estimate $\gamma_{0}$ in the next subsection, later we will obtain $\delta_{0}=\delta\left(t_{0}\right)$ from supernovae data, and finally, all the other parameters will be derived from these two quantities.

3.1. Cosmological Blueshift and the Pioneer Anomaly. In Section 2, we have summarized all the fundamental expressions of our cosmology and outlined the reasons why we consider the $\mathbf{k}=-1$ solution as a possible description of the evolution of the Universe. Although this solution can explain the observed cosmological redshift, it has an additional new feature. It requires the existence of a blueshift region in the immediate vicinity of our current space-time position in the Universe. 
This could be a serious setback for our model, since we normally do not observe blueshift of nearby astrophysical objects except for the one caused by the peculiar velocities of nearby galaxies, which is presumably due to standard Doppler shift. However, experimental evidence has recently accumulated regarding a possible local region of blueshift, related to the so-called Pioneer anomaly [21-31].

This is a small anomalous frequency drift, actually a blueshift, which was observed analyzing the navigational data from the Pioneer 10/11 spacecraft, received from distances between 20 and 70 astronomical units from the sun, while exploring the outer solar system. This "blueshifted" frequency is uniformly changing at a rate of $\dot{\nu}_{P} \cong 6 \times 10^{-9} \mathrm{~Hz} / \mathrm{s}$ and can be interpreted as a constant sunward acceleration, reported as $a_{P}=-(8.74 \pm 1.33) \times 10^{-8} \mathrm{~cm} / \mathrm{s}^{2}($ radial inward acceleration) or as a "clock acceleration" $a_{t} \equiv a_{P} / c=$ $-(2.92 \pm 0.44) \times 10^{-18} \mathrm{~s} / \mathrm{s}^{2}$, resulting in a frequency drift of about $1.5 \mathrm{~Hz}$ every 8 years [22].

Preliminary findings indicate the possibility of detecting such anomaly also in the radiometric data from other spacecraft traveling at the outskirts of the solar system, such as the Galileo and Ulysses missions [22]. These discoveries have prompted an extended reanalysis of all the historical navigational data from these space missions, which is currently underway $[25-28,32]$, in order to determine additional characteristics of the anomaly, such as its precise direction, the possible temporal evolution, and its dependence on heliocentric distance. Future-dedicated missions are also being planned [33-35] to test directly this puzzling phenomenon.

Meanwhile, the origin and nature of this anomaly remains unexplained; all possible sources of systematic errors have been considered [22-24, 27, 28, 36], but they cannot account for the observed effect. Possible physical origins of the signal were studied, including dark matter, modified gravity, or other nonconventional theories, but no satisfactory explanation has been found so far (see details in $[22,29,30,32])$. On the contrary, we can analyze the Pioneer anomaly with the model outlined in the previous sections and use the data reported above to estimate the local values of our cosmological parameters.

The phenomenology of the Pioneer anomaly is related to the exchange of radiometric data between the tracking station on Earth (Deep Space Network-DSN) and the spacecraft, using S-band Doppler frequencies (in the range $1.55-5.20 \mathrm{GHz}$ ). More precisely, an uplink signal is sent from the DSN to the spacecraft at $2.11 \mathrm{GHz}$, based on a very stable hydrogen maser system, the Pioneer then returns a downlink signal at a slightly different frequency of about $2.29 \mathrm{GHz}$, to avoid interference with the uplink signal. This is accomplished by an S-band transponder which applies an exact and fixed turn-around ratio of 240/221 to the uplink signal.

This procedure, known as a two-way Doppler coherent signal, allows for very precise tracking of the spacecraft since the returning signal is compared to the original one, as opposed to a one-way Doppler signal (fixed signal source on spacecraft, whose frequency cannot be directly monitored for accuracy). This type of tracking system and the navigational capabilities of the Pioneer spaceship (spinstabilized spacecraft and power source of special design) allowed for a great acceleration sensitivity of about $10^{-8} \mathrm{~cm} / \mathrm{s}^{2}$, once the influence of solar-radiation-pressure acceleration decreased below comparable levels (for distances beyond about $20 \mathrm{AU}$ from the Sun).

After a time delay of a few minutes or hours, depending on the distance involved, the DSN station acquires the downlink signal, and any difference from its expected frequency is interpreted as a Doppler shift due to the actual motion of the spacecraft. Modern-day deep space navigational software can also predict with exceptional precision the expected frequency of the signal returned from the Pioneer, which should coincide with the one observed on Earth. On the contrary, a discrepancy was found, corresponding to the values indicated above, whose origin cannot be traced to any systematic effect due to either the performance of the spacecraft or the theoretical modeling of its navigation.

Moreover, the signal analysis performed so far [23, 26] indicates an almost constant value of the anomalous acceleration or frequency shift reported above (temporal and space variation of $a_{P}$ within $10 \%$ ), over a range of heliocentric distances $\sim 20-70 \mathrm{AU}$, and possibly at even closer distances $\lesssim 10$ AU.

The Pioneer phenomenology corresponds exactly to the simplest experiment we might conceive to check the validity of our model. In principle, it would be sufficient to set up a lab experiment in which we emit some radiation of known wavelength $\lambda\left(t_{0}\right)$ at time $t_{0}$, keep this radiation from being absorbed for a long enough time, and then compare its wavelength $\lambda\left(t_{0}\right)$ with the radiation emitted by the same source at a later time $\lambda\left(t_{1}\right)$, with $t_{1}>t_{0}$. In our model, we would expect the two wavelengths to be different and, if we are already in a phase of universal contraction as illustrated by the red-solid curve of Figure 5 in our paper I [7], we would have $\lambda\left(t_{0}\right)<\lambda\left(t_{1}\right)$. In terms of frequencies, $v\left(t_{0}\right)>$ $v\left(t_{1}\right)$, that is, the radiation from time $t_{0}$ would appear to be blueshifted, compared to the same radiation emitted by the same source at a later time $t_{1}$. We will now proceed to interpret the Pioneer anomaly in a similar way, but we will return in Section 4 on the feasibility of detecting wavelength variation in lab experiments.

It is useful to introduce a new time coordinate $\bar{t}$ : let $\bar{t}=0$ be the time at which the radiometric signal is sent from Earth to the spacecraft, which is then received at time $\bar{t}$ and immediately retransmitted down to the DSN, arriving back on Earth at time $2 \bar{t}$. In the standard analysis, the model for the signal coming back to Earth is based on the relativistic Doppler effect. We will denote as $v_{\bmod }$ the frequency of the expected signal following this model. Its ratio with the reference frequency $v_{\text {ref }}$ of the signal (the uplink frequency of about $2.11 \mathrm{GHz}$, since we consider the two-way system) is therefore given by the standard relativistic Doppler formula (see (2.2.2) in [15])

$$
\frac{v_{\text {mod }}}{v_{\text {ref }}}=\frac{\sqrt{1-\mathbf{v}^{2} / c^{2}}}{1+v_{r} / c} \simeq 1-\frac{v_{r}}{c},
$$

where $v_{r}$ is the source radial velocity, and the approximation on the right-hand side holds to first order in $v_{r} / c$. 
For the case of the Pioneer spacecraft, we use $v_{r}=$ $2 v_{\text {mod }}(\bar{t})$, where $v_{\text {mod }}(\bar{t})$ is the expected velocity of the spacecraft, according to the theoretical navigation model, at time $\bar{t}$, when the spaceship receives and immediately retransmits the signal. The factor of two arises from the double Doppler shift involved (two-way system). With this radial velocity, (31) to first order in $v_{r} / c$ becomes

$$
v_{\text {mod }}(\bar{t}) \simeq v_{\text {ref }}\left[1-\frac{2 v_{\text {mod }}(\bar{t})}{c}\right],
$$

and this frequency should be observed with high precision, due to the reported excellent navigational control of the spacecraft.

The anomaly comes from observing a different frequency $v_{\text {obs }}(\bar{t})>v_{\text {mod }}(\bar{t})$, involving an additional unexplained blueshift. Over the range of the observed anomaly, the frequency difference is reported as

$$
\begin{gathered}
\Delta v=v_{\mathrm{obs}}(\bar{t})-v_{\bmod }(\bar{t}) \simeq \dot{v}_{P} 2 \bar{t}, \\
\dot{v}_{P} \cong 6 \times 10^{-9} \mathrm{~Hz} / \mathrm{s} .
\end{gathered}
$$

We point out here that the cited references adopt a rather confusing "DSN sign convention" for the frequency difference in (33) (see $[23,26]$ and reference [36] of [21]), resulting in a sign change in most of their equations. We prefer to use here our definition of $\Delta v$ as given in the previous equation.

An alternative way of reporting the anomaly is the following. As in (32), we can also write the observed frequency to first order in $v_{r} / c$ as

$$
v_{\mathrm{obs}}(\bar{t}) \simeq v_{\text {ref }}\left[1-\frac{2 v_{\mathrm{obs}}(\bar{t})}{c}\right],
$$

where the "observed" velocity of the spacecraft always refers to the time of interest $\bar{t}$. Combining together these last three equations, we write the frequency difference as

$$
\Delta v=-2 \frac{v_{\mathrm{ref}}}{c}\left[v_{\mathrm{obs}}(\bar{t})-v_{\bmod }(\bar{t})\right]
$$

We then multiply and divide the last equation by $\bar{t}$, so that we can introduce $a_{P}=\left[v_{\text {obs }}(\bar{t})-v_{\text {mod }}(\bar{t})\right] / \bar{t}$, the residual Pioneer acceleration of unknown origin. This is the change of velocity over time $\bar{t}$ and not over the double time $2 \bar{t}$ as in (33). With this residual acceleration, the Pioneer anomaly is usually reported as

$$
\begin{gathered}
\Delta v=-2 \frac{\nu_{\text {ref }}}{c} a_{P} \bar{t}, \\
a_{P}=-(8.74 \pm 1.33) \times 10^{-8} \frac{\mathrm{cm}}{\mathrm{s}^{2}},
\end{gathered}
$$

where the negative sign of the residual acceleration indicates its sunward direction; therefore, we have a positive frequency shift corresponding to a local unexplained blueshift of radiation emitted by the spacecraft.
Given our discussion in the preceding sections, we can now explain (36) or (33) with our new interpretation. The Pioneer data represent the equivalent of a local measurement of the current blueshift predicted in Section 2.1; therefore, they can be used to find the value of the parameter $\gamma$ at the time when the Pioneer radiation was emitted (a few years ago), but this can be considered to be the current value, due to the slow variation of the cosmological parameters.

Let us consider the current time $t_{0}$ as the time of arrival of the Pioneer radiation on Earth. The uplink signal was therefore sent at $t=t_{0}-2 \bar{t}$ and retransmitted by the spacecraft, as a downlink signal, at $t=t_{0}-\bar{t}$. We need to reinterpret (32) and (34) as, in our view, the anomaly is not due to a change in velocity of the spacecraft (we assume $\left.v_{\text {mod }}(\bar{t})=v_{\text {obs }}(\bar{t})=v(\bar{t})\right)$, but just related to a shift of the reference frequency. The reference frequency in (34) is the one emitted in the past $\nu_{\text {ref }}=v\left(t=t_{0}-2 \bar{t}\right)$, while the reference frequency in (32) is the one at current time $\nu_{\text {ref }}=$ $v\left(t=t_{0}\right)$, so these two equations are modified, respectively, as follows

$$
\begin{gathered}
v_{\mathrm{obs}}(\bar{t}) \simeq v\left(t=t_{0}-2 \bar{t}\right)\left[1-\frac{2 v(\bar{t})}{c}\right], \\
v_{\bmod }(\bar{t}) \simeq v\left(t_{0}\right)\left[1-\frac{2 v(\bar{t})}{c}\right] .
\end{gathered}
$$

Following (33), the frequency difference, to first order in $v / c$, is now

$$
\begin{aligned}
\Delta v & =\left[v(t)-v\left(t_{0}\right)\right]\left[1-\frac{2 v(\bar{t})}{c}\right] \\
& =v(t)\left[1-\frac{R\left(t_{0}\right)}{R(t)}\right]\left[1-\frac{2 v(\bar{t})}{c}\right] \simeq \dot{\nu}_{P}\left(t_{0}-t\right),
\end{aligned}
$$

having used our fundamental (37) from paper I [7] and also $\left(t_{0}-t\right)=2 \bar{t}$. We can simplify the above equation by introducing additional approximations. From the second line of (2), we have $1-R\left(t_{0}\right) / R(t)=1-\left\{\cosh \left[\sqrt{|k|} c\left(t_{0}-t\right)\right]-\right.$ $\left.(\gamma / 2 \sqrt{|k|}) \sinh \left[\sqrt{|k|} c\left(t_{0}-t\right)\right]\right\} \cong(\gamma / 2) c\left(t_{0}-t\right)$, since we can assume $\sqrt{|k|} c\left(t_{0}-\mathrm{t}\right) \ll 1$ (The time delay for a two-way Pioneer signal at the maximum distance of $70 \mathrm{AU}$ is about 20 hours; we can assume $\sqrt{|k|} \sim \gamma \sim 10^{-28}-10^{-30} \mathrm{~cm}^{-1}$, therefore $\sqrt{|k|} c\left(t_{0}-t\right) \sim 10^{-13}-10^{-15} \ll 1$.). The reference frequency is $\nu_{\text {ref }}=v(t) \cong v\left(t_{0}\right)=2.11 \mathrm{GHz}$, corresponding to the original uplink frequency (In some of the references cited, the reference frequency $\nu_{\text {ref }}$ is taken as the downlink frequency of $2.29 \mathrm{GHz}$, since the downlink signal is compared directly with this value. We prefer to use the uplink value, since this is the frequency used at the original time $t=t_{0}-2 \bar{t}$.), and we can also neglect the ratio $2 v(\bar{t}) / c \ll 1$, since the typical Pioneer speed in the outer solar system was $v \approx 12 \mathrm{~km} / \mathrm{s}[23]$. With these approximations, the last equation becomes

$$
\Delta v \cong v_{\mathrm{ref}}\left[\frac{\gamma}{2} c\left(t_{0}-t\right)\right] \cong \dot{\nu}_{P}\left(t_{0}-t\right)
$$


The clock acceleration $a_{t}$, the Pioneer acceleration $a_{P}$, the frequency shift $\dot{\nu}_{P}$, and the reference frequency $\nu_{\text {ref }}$ are all related together by

$$
a_{t}=\frac{a_{P}}{c}=-\frac{\dot{v}_{P}}{v_{\text {ref }}},
$$

which follows from (33) and (36). Combining these last two equations and using the reported values of the Pioneer anomaly, we can finally obtain our estimate of the current local value of the cosmological parameter $\gamma$,

$$
\gamma_{0}=\gamma\left(t_{0}\right) \cong \frac{2}{c} \frac{\dot{\nu}_{P}}{\nu_{\mathrm{ref}}}=-\frac{2}{c^{2}} a_{P}=-\frac{2}{c} a_{t}=1.94 \times 10^{-28} \mathrm{~cm}^{-1},
$$

which represents the best estimate of $\gamma$ at our current time (although the Pioneer data are a few years old). (A more complete discussion of the derivation of our cosmological parameters, based only on the Pioneer anomaly, can be found in our latest work [37].) This is the value which was quoted in Section 2.2 and led to our evaluation of the Hubble constant. (Since the discovery of the Pioneer anomaly, many researchers have noticed the numerical "coincidence" between the Hubble constant and the value of the Pioneer acceleration $a_{P}$ divided by $c$ and proposed many different explanations for this. This coincidence is even more striking if one uses the value cited in [23] as the experimental value for Pioneer 10 data before systematics, $a_{P}=-7.84 \times$ $10^{-8} \mathrm{~cm} \mathrm{~s}^{-2}$, thus obtaining $H_{0}=80.7 \mathrm{~km} \mathrm{~s}^{-1} \mathrm{Mpc}^{-1}$ and $\gamma_{0}=1.74 \times 10^{-28} \mathrm{~cm}^{-1}$. Although this choice would result in a value of the Hubble constant closer to standard cosmology evaluations, we prefer to base all our analysis on the usually quoted value of $a_{P}$ as in (36).)

In the previous equation, we connected $\gamma$ to the measured Pioneer acceleration $a_{P}$ (or the clock acceleration $a_{t}$ ) simply because such was the way these data were reported in the literature cited. However, it should be clear from the discussion above that we explain the Pioneer anomaly in terms of our cosmological-gravitational blueshift (equivalent in a way to the clock acceleration mentioned above). In this view, there is no real dynamic acceleration of the Pioneer spacecraft (or of any other object in the solar system) oriented toward the sun, due to some new gravitational force or modification of existing gravity. In fact, $a_{P}=$ $\left[v_{\mathrm{obs}}(\bar{t})-v_{\text {mod }}(\bar{t})\right] / \bar{t}=0$ in our analysis, since we assume there is no difference between the two velocities $v_{\mathrm{obs}}(\bar{t})$ and $v_{\text {mod }}(\bar{t})$.

In this way, we overcome the objection, reported, for example, in [23], that "the anomalous acceleration is too large to have gone undetected in planetary orbits, particularly for Earth and Mars," since "NASA's Viking mission provided radio-ranging measurements [38] to an accuracy of about $12 \mathrm{~m}$," which should have shown the effect of the anomalous acceleration on the orbits of these two planets.

In fact, there cannot be any anomalous acceleration for the Earth or Mars if we measure distances with round-trip time of flight of radio signals transmitted from Earth to the Viking spacecraft on the Mars surface [39]. On the contrary, we would observe a similar effect for Earth, Mars, or any other object in the solar system, if we were to study its motion through Doppler frequency ranging, because of the intrinsic differences in frequency or wavelength for light emitted at different space-time positions, due to our new cosmological effects.

We will return on this difference between experimental techniques for estimating distances in a later section. We conclude this section by noting that if the Pioneer anomaly is indeed caused by our new cosmological effects and not by modification of the gravity from the sun, the "direction" of the anomalous "acceleration" should be pointing towards the terrestrial observer and not towards the sun. (Since our cosmology is based on (2), which was derived in paper I for regions in space far away from mass sources, i.e., independent of the location and size of massive objects in the Universe, the anomalous acceleration does not need to point in the direction of a massive source, such as the Sun, the center of our galaxy, or others. The effect should be seen in the same way from every point of observation in the Universe, and the observed anomalous acceleration would seem to be pointing simply towards the point of observation (Earth, in this case).) This is currently being studied (see [26] for details) by using data from a period of time when the spacecraft was much closer to the Earth and the sun, so that a clear direction of the anomaly can be determined, but no results from this new analysis are available yet.

3.2. Kinematical Conformal Cosmology and Type Ia Supernovae. The determination of $\delta_{0}$ can be done with the powerful astrophysical tool represented by type Ia supernovae (SNe Ia) used as standard candles (see "The High-Z SN Search" website [40], or the "High Redshift Supernova Search Supernova Cosmology Project" web page [41] for an introduction to the topic, see also [42-44] for reviews on the subject). Since the original discoveries made by these two leading groups $[45,46]$, the observational data were recently expanded to the so-called "gold" and "silver" sets ([47, 48] and references therein) including the highest redshift supernovae known, at $z \gtrsim 1.25$. From these data, we will determine the value of $\delta_{0}$ and then obtain all the other parameters.

The use of type Ia SNe as standard candles involves the revision of the concept of luminosity distance and related quantities, in view of our alternative cosmology. The observational techniques must also be carefully considered, since they are based on the current standard cosmology. We have seen in the preceding section how to generalize the luminosity distance in line with our new interpretation; we will now apply those concepts to the case of type Ia SNe.

Equations (19)-(24) have shown that quantities such as the absolute luminosity of standard candles and others depend on their cosmological location, simply described by the redshift parameter $z$. We want to stress once more that in our new interpretation all the characteristic quantities of emission and absorption of radiation, such as frequencies, wavelengths, time intervals between radiative events, and energies, are intrinsically dependent on the space-time location of the events being observed. No receding motion 
is needed, and no cosmological Doppler shift is inducing redshifts in the observed spectra, but care is to be used to evaluate the different quantities at the space-time location of interest, and the proper corrections will enter the formulas only when we compare the same quantity at different cosmological locations.

For example, in our view, the "absolute" luminosity of a standard candle at position $\mathbf{r}$ emitting light at a past time $\mathbf{t}$ would be measured differently by two observers, one placed at the origin $\mathbf{r}=0$ observing the radiation at current time $\mathbf{t}_{0}$ and the other at the source $\mathbf{r}$ observing the radiation at time $\mathbf{t}$. (The absolute luminosity is the total power emitted by the source. Obviously only apparent luminosities, i.e., power per unit area, can be measured by observers far away from the source. The absolute luminosity $L_{z}$ therefore refers to an ideal measurement, as if we could measure all the energy flowing through a spherical surface of radius $\mathbf{r}$, centered around the source.) Following our notation mentioned above, the former observer would measure the luminosity $L_{z}$, which was used in the previously mentioned equations to derive the luminosity distance. The latter, observing the radiation at the source, would measure what we denote by $L_{0}$, that is, the equivalent of placing the standard candle near the origin and observing it at our current time. The two luminosities $L_{z}$ and $L_{0}$ are then related by (21).

To further clarify this issue with an example, a standard candle of "intrinsic" total luminosity $L_{0}=1 \mathrm{erg} / \mathrm{s}$ and "intrinsic" spectral color, for example, a "blue" candle, will always have the same luminosity and always show its blue color when seen by an observer placed near the candle, employing the appropriate local units of space, time, energy, and so forth. On the contrary, when placed at some cosmological distance corresponding to some definite $z$ value (say $z=1$, e.g.), it would be observed as a "red" candle and having a different luminosity $L_{z}=f(1+z) L_{0} /(1+z)$ following (21), using units proper to the observer at position $\mathbf{r}=0$ and time $\mathbf{t}_{0}$. All these effects are just due to the different intrinsic units used by the two observers, as related to (19), and are not in any way connected to relativistic Doppler shifts, which might be only additional corrections due to the peculiar motion of the source relative to the observer.

The only missing piece in our luminosity definition is the explicit expression of the function $f(1+z)$. We might expect this function to be related to the usual $(1+z)$ factor as discussed before, but we have no reason to assume a simple dependence such as the one followed by space-time intervals in (19). In choosing the form for the function $f(1+z)$, we are guided by the following considerations. The current theory of standard candles, as already remarked before, considers a reference distance $d_{\mathrm{ref}}=10 \mathrm{pc}$, at which the apparent luminosity of the candle is taken as the absolute luminosity. This leads to the standard expression of the luminosity distance as in (17). Since our luminosity $L_{z}$ depends on $(1+z)$, it seems more correct to place the candle at a position where $z=0$, in order to estimate its "absolute" luminosity. Instead of having the candle at the origin of the space coordinates (a rather impracticable choice if the candle is a supernova), we can place it at a distance $d_{\mathrm{rs}}=\mathbf{R}_{0} \mathbf{r}_{\mathrm{rs}}=$ $\mathbf{R}_{0}\left(2 \delta_{0} /\left(1-\delta_{0}^{2}\right)\right)$, at the beginning of the "redshift region," where it is also $z=0$, as discussed at length in our first paper. In other words, there are only two positions where a standard candle's luminosity is unaffected by the universal conformal stretching: one is at the origin and the other, more conveniently used as a reference distance, is this special distance $d_{\mathrm{rs}}$, which also depends on the current value $\delta_{0}$ of our fundamental parameter.

The second hypothesis that will be made is on the form of the function $f(1+z)$. In this, we are guided again by the classic definition of the luminosity distance, based on the inverse square law. In fact, we propose a generalization of the original inverse square law $l=L / 4 \pi d^{2}$ which assumed $L$ to be invariant, to a generalized form $l=L_{0} / 4 \pi d^{a}$ where now $L_{0}$ is measured near the source, therefore constant by definition, but we generalize the power dependence on the distance to account for the luminosity variations with $z$. Since this form of the revised inverse-square law would be dimensionally incorrect for $a \neq 2$, we further refine it by including the reference distance $d_{\text {ref }}=d_{\text {rs }}$ as follows:

$$
\begin{gathered}
l=\frac{L_{z}\left(t_{z}\right)}{4 \pi d_{L}^{2}}=\frac{L_{0}\left(t_{0}\right)}{4 \pi d_{L}^{2}\left(d_{L} / d_{\mathrm{ref}}\right)^{a}}, \\
L_{z}\left(t_{z}\right)=\frac{L_{0}\left(t_{0}\right)}{\left(d_{L} / d_{\mathrm{ref}}\right)^{a}}, \\
f(1+z)=(1+z) \frac{L_{z}\left(t_{z}\right)}{L_{0}\left(t_{0}\right)}=\frac{(1+z)}{\left(d_{L} / d_{\mathrm{ref}}\right)^{a}} \\
=(1+z)\left[\frac{2 \delta_{0}}{\delta_{0}(1+z)+\sqrt{(1+z)^{2}-\left(1-\delta_{0}^{2}\right)}}\right]^{a} .
\end{gathered}
$$

In the equation above, we show in the first line our "inverse-power law" generalization of the apparent luminosity-distance relationship, with an exponent $a$ to be determined by fitting the type Ia SNe data. This generalization implies the dependence on $z$ of the absolute luminosity $L$, as given in the second line, and by comparison with the (21), it determines the form of the unknown function $f(1+z)$, as given in the last line of (42).

We want to emphasize here that our choice of the function $f(1+z)$ or of the generalized "inverse-power law" is just an educated guess, which will lead to a good fit of the $\mathrm{SNe}$ data in the following. At this stage, we cannot justify it on the basis of our kinematical conformal cosmology. Therefore, going back to our (19) which details the scaling properties of units of length, time, and mass, while the first two lines are fundamentally based on our new interpretation, the last expression with the function $f(1+z)$ of $(42)$ is currently our best hypothesis but might need to be revised in the future.

In any case, assuming our current hypothesis to be a reasonable one, we can finally obtain the equivalent of (17), which will express the distance modulus $\mu(z)$ directly as 
a function of the redshift parameter. Combining together (18), (22), and (42), we obtain

$$
\begin{aligned}
\mu_{\mathrm{bol}}\left(z ; a, \delta_{0}\right)= & m_{\mathrm{bol}}\left(z, t_{z}\right)-M_{\mathrm{bol}}\left(z=0, t_{0}\right) \\
= & -2.5 \log _{10}\left[l_{\mathrm{bol}}\left(z, t_{z}\right) / l_{\mathrm{bol}}\left(z=0, t_{0}\right)\right] \\
= & 2.5(2+a) \log _{10}\left(d_{L} / d_{\mathrm{ref}}\right) \\
= & 2.5(2+a) \log _{10} \\
& \times\left[\frac{\delta_{0}(1+z)+\sqrt{(1+z)^{2}-\left(1-\delta_{0}^{2}\right)}}{2 \delta_{0}}\right] .
\end{aligned}
$$

In this equation, we have indicated that all magnitudes, luminosities, and so forth, refer to bolometric quantities, that is, are measured over all wavelengths of emitted radiation. We will consider later the effect of observing this radiation with particular filters. We have also indicated the time dependence of the observed quantities, where as mentioned before $t_{z}=$ $t_{0}(1+z)$. This is due to the fact that type Ia SNe has a temporal evolution [17-20] and the luminosities are usually referred to the peak values. The time at which a Supernova reaches its peak luminosity differs if observed near the source $\left(t_{0}^{\text {peak }}\right)$ or if it is seen from a cosmological distance, in which case $t_{z}^{\text {peak }}=t_{0}^{\text {peak }}(1+z)$.

In (43), the (bolometric) distance modulus $\mu_{\mathrm{bol}}\left(z ; a, \delta_{0}\right)$ is an explicit function of the redshift variable $z$ and of the two parameters $a$ and $\delta_{0}$, whose values will be determined by fitting this formula to the experimental data of type Ia SNe. Our treatment therefore parallels the standard cosmology model where usually $\mu_{\text {bol }}\left(z ; \Omega_{M}, \Omega_{\Lambda}\right)$ is a function of the density parameters $\Omega_{M}, \Omega_{\Lambda}$. The comparison with experimental observations is usually carried out by fitting these expressions to the measured distance moduli $\mu_{\text {bol }}$, for several types of Ia SNe as observed by the most recent surveys $[47,48]$.

The determination of the bolometric magnitudes from the astrophysical measurements is quite complex, involving conversions from observations performed in precise wavelength bands ( $U, B, V, R, I$ filters, for ultraviolet, blue, visible, red, and infrared bands resp.) to the total (bolometric) luminosities and corresponding magnitudes. This involves precise "K-corrections" to transform the observations in the different bands to the bolometric quantities, plus other corrections involving the extinction of the $\mathrm{SNa}$ light in the host galaxy as well as in our own galaxy, resulting in a complex procedure which is also able to discriminate the overall "quality" of the candidate Supernova. A more detailed analysis of some of these procedures will be given in the appendix, in order to ascertain that they are consistent with our new cosmological interpretation and with (43).

We have considered the best current compilation of existing data, given by the $292 \mathrm{SNe}$ of the "gold-silver set" taken from [48, Table 4] and also available in a machinereadable form in [49]. In this table, as well as in similar data compilations, the normalization of the distance moduli is usually arbitrary, since only the relative distances are needed to obtain the dynamical cosmological parameters, such as $\Omega_{M}, \Omega_{\Lambda}$. The overall normalization of the data is linked to the value of the Hubble constant, but this is usually treated as a nuisance parameter in the fitting procedure, therefore not explicitly determined.

This approach follows from the standard cosmology definition of the luminosity distance [50] as a function of $z$, of the density parameters $\Omega_{M}, \Omega_{\Lambda}$, and of the Hubble constant $H_{0}$,

$$
\begin{aligned}
& d_{L}\left(z ; \Omega_{M}, \Omega_{\Lambda}, H_{0}\right) \\
& =\frac{c(1+z)}{H_{0} \sqrt{|\kappa|} S} \\
& \quad \times\left\{\sqrt { | \kappa | } \int _ { 0 } ^ { z } \left[\left(1+z^{\prime}\right)^{2}\left(1+\Omega_{M} z^{\prime}\right)\right.\right. \\
& \left.\left.\quad-z^{\prime}\left(2+z^{\prime}\right) \Omega_{\Lambda}\right]^{-1 / 2} d z^{\prime}\right\},
\end{aligned}
$$

where for $\Omega_{M}+\Omega_{\Lambda}>1, S(x) \equiv \sin (x)$ and $\kappa=1-\Omega_{M}-\Omega_{\Lambda}$; for $\Omega_{M}+\Omega_{\Lambda}<1, S(x) \equiv \sinh (x)$ and $\kappa$ as above; for $\Omega_{M}+$ $\Omega_{\Lambda}=1, S(x) \equiv x$ and $\kappa=1$.

Since $d_{L}$ is inversely proportional to $H_{0}$, following (17), the standard expression of the distance modulus becomes:

$$
\begin{aligned}
\mu(z) & =m(z)-M=5 \log _{10} d_{L}\left(z ; \Omega_{M}, \Omega_{\Lambda}, H_{0}\right)+25 \\
& =5 \log _{10} \mathcal{D}_{L}\left(z ; \Omega_{M}, \Omega_{\Lambda}\right)-5 \log _{10}\left(H_{0}\right)+25,
\end{aligned}
$$

introducing the so-called "Hubble constant-free" luminosity distance $\mathscr{D}_{L}\left(z ; \Omega_{M}, \Omega_{\Lambda}\right) \equiv H_{0} d_{L}$. The term containing the Hubble constant is then usually summed together with $M$ and the other constants in the previous equation and then integrated upon in the standard fitting procedure.

However, as noted in [49], the data of the latest "goldsilver" sets can be reconciled with the latest CepheidSNe-based Hubble constant value [12] $\left(H_{0}=(73 \pm 6.4)\right.$ $\mathrm{km} \mathrm{s}^{-1} \mathrm{Mpc}^{-1}$ ) by subtracting $0.32 \mathrm{mag}$ from all distance moduli in [49, Table 4]. By performing this adjustment, the distance moduli $\mu$ become consistent with $H_{0} \cong$ $73 \mathrm{~km} \mathrm{~s}^{-1} \mathrm{Mpc}^{-1}$, which is however different from our value in (9), $H_{0} \cong 89.7 \mathrm{~km} \mathrm{~s}^{-1} \mathrm{Mpc}^{-1}$.

Since standard cosmology assumes that the luminosity distance $d_{L}$ is inversely proportional to $H_{0}$ as noted above, it is easy to check that subtracting from all data another common value equal to $5 \log _{10}(89.7 / 73) \cong 0.45$, related to the ratio of the Hubble constants reported above, will bring all the distance moduli in line with our preferred value of the Hubble constant.

Therefore, we have performed this "double correction," subtracting the factor $0.32+0.45=0.77$ from all the distance moduli of the "gold-silver" sets, to bring them in line with our assumptions. This is the best option we have to fix the normalization of the existing data in the literature, since we do not have access to the normalization algorithm used by the $\mathrm{SNe}$ groups (see also the discussion in the appendix).

We have plotted these "double-corrected" distance moduli $\mu$ as a function of the observed redshift $z$ in Figure 1 (yellow circular points for "gold" data, grey triangular points for "silver" $\mathrm{SNe}$ ). In the same figure, we fit the expression in (43) to the experimental data, choosing the "gold set" only 


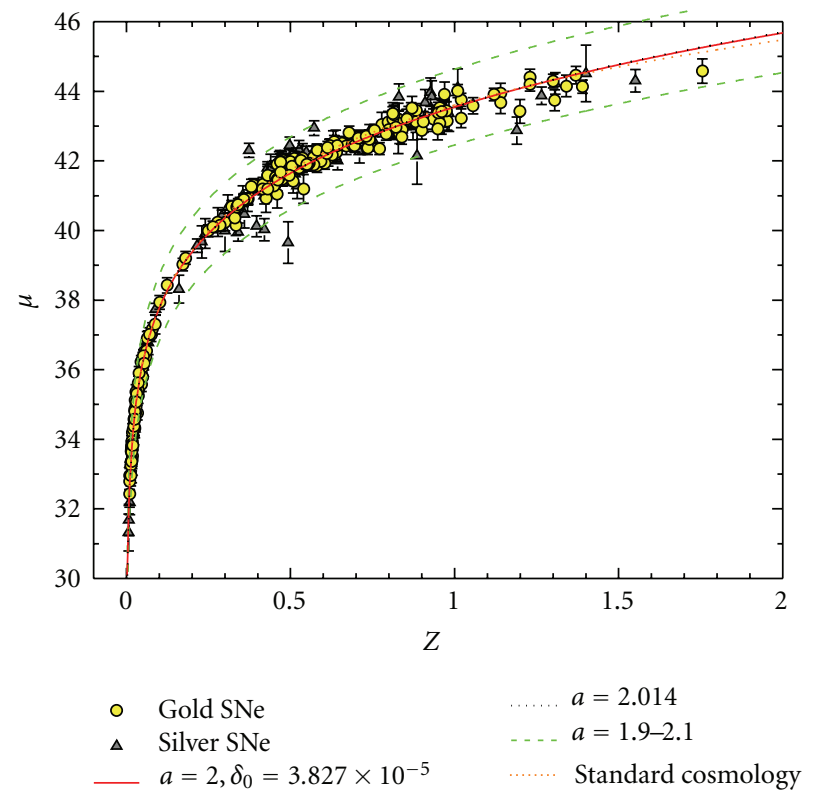

FIgURE 1: Data from type Ia SNe "gold-silver sets" [48] are fitted with (43). Our fits show a remarkably good quality $\left(\chi^{2}=0.053\right.$; $R^{2}=0.996$ ) for both a variable $a$ (black-dotted curve) and for a fixed $a=2$ (solid red curve). In this latter case, the best fit parameter for delta is $\delta_{0}=3.827 \times 10^{-5}$. Also shown (dashed green curves) is the range of our fitting curves for $a=1.9-2.1$ and the Standard Cosmology curve (in orange, short dashed).

for our fit, due to the greater reliability of these data over the "silver set." (We have performed similar fits including both "gold" and "silver" data for completeness. We obtained basically the same results as in the case of "gold" only, with a slightly worse statistical quality of the fit.) Our fit for the "gold" SNe has a good statistical quality $\left(\chi^{2}=0.0534 ; R^{2}=\right.$ $0.996)$, and we obtain the best fit parameters as follows:

$$
\begin{gathered}
a=2.014 \pm 0.018 \\
\delta_{0}=(3.951 \pm 0.167) \times 10^{-5} .
\end{gathered}
$$

The resulting fit is shown as a black-dotted curve in the figure. Given our hypothesis of an "inverse power law," our fit seems to suggest an integer value $a=2$ for the exponent of the factor $\left(d_{L} / d_{\text {ref }}\right)$ in (42). If we perform a new fit of the same data, keeping $a=2$ fixed, we obtain the continuous red curve in Figure 1 (virtually equivalent to the black-dashed one) and a slightly different value for $\delta_{0}$,

$$
\begin{gathered}
a=2, \\
\delta_{0}=(3.827 \pm 0.014) \times 10^{-5} .
\end{gathered}
$$

Since this is also a good-quality fit of the data $\left(\chi^{2}=\right.$ $\left.0.0533 ; R^{2}=0.996\right)$, we are inclined to consider the values given in (47) as our best estimates of these parameters. In particular, we will use in the following our inverse power law with an integer exponent $a=2$. In Figure 1, we also plot the curves (green dashed) for $a=1.9-2.1$, keeping $\delta_{0}$ as in (47), to show how our fitting solution depends on the parameter $a$. It can be seen also that the majority of the experimental points lie within this range, confirming our hypothesis of $a \simeq 2$.

We also show for comparison the standard cosmology fitting curve (orange, short dashed), following (44)-(45), for a flat Universe with $\Omega_{M}=0.27, \Omega_{\Lambda}=0.73$, and $H_{0}=$ $89.7 \mathrm{~km} \mathrm{~s}^{-1} \mathrm{Mpc}^{-1}$ to bring it in line with our value of the Hubble constant. Our preferred fit (in red solid) is practically equivalent to the standard cosmology curve, except for a slight difference at highest redshift values, thus showing that our model can produce Hubble plots of the same quality of those obtained by current cosmology, but without requiring any dark matter or dark energy contribution.

Our analysis of type Ia SNe therefore confirms the applicability of the kinematical conformal cosmology to standard candle luminosity measurements and proposes a small positive value $\delta_{0} \simeq 3.83 \times 10^{-5}$ for our fundamental cosmological parameter, as noted previously in this work and also in paper I.

We have to mention again that the set of "gold-silver" SNe data and particularly the distance moduli $\mu(z)$ from [48] that we used in our analysis were originally obtained through a rather complex calibration algorithm (called MLCS2k2, see $[47,48]$ for complete details) which takes into consideration the wavelength bands being used ( $U, B, V, R, I$, etc.) and related K-corrections, the current value of the Hubble constant, the extinction and reddening effects, the zero point luminosity calibration, and absolute magnitude of type Ia SNe.

This calibration method originated in the early papers of the two leading groups, as the "multicolor light-curve shape" (MLCS- $[51,52])$ then was revised into the latest MLCS2k2, and the template fitting method- $\Delta m_{15}$ [53]. In these early works, it was still possible to find a step-by-step description of the methods being used and the values of almost all the corrections employed, in order to check our normalization procedure.

On the contrary, in the latest papers based on the MLCS2k2 method [12, 47, 48], which produced the "goldsilver set" of distance moduli that we used in Figure 1, the complexity of the parametrization and fitting procedure makes the comparison with our luminosity normalization procedure very difficult. While we will analyze the Kcorrections in the appendix, we make no attempt to revise the other corrections and normalization procedures for the distance moduli (except for the "double correction" we used above to bring the data in line with our preferred value of the Hubble constant).

In Figure 2, we present the same data and similar fitting curves as in Figure 1, but in the form of residual values $\Delta \mu$, where the baseline is represented by our fit (in solid red) with the values of (47). It can be seen again how most of the $\mathrm{SNe}$ data fall within the $a=1.9-2.1$ band.

Before proceeding to study the other cosmological parameters, we want to analyze briefly the low-z case and the related fit to supernova data, to confirm the feasibility of our approach also at low redshift. In standard cosmology, the expression of the luminosity distance as a function of $z$, 


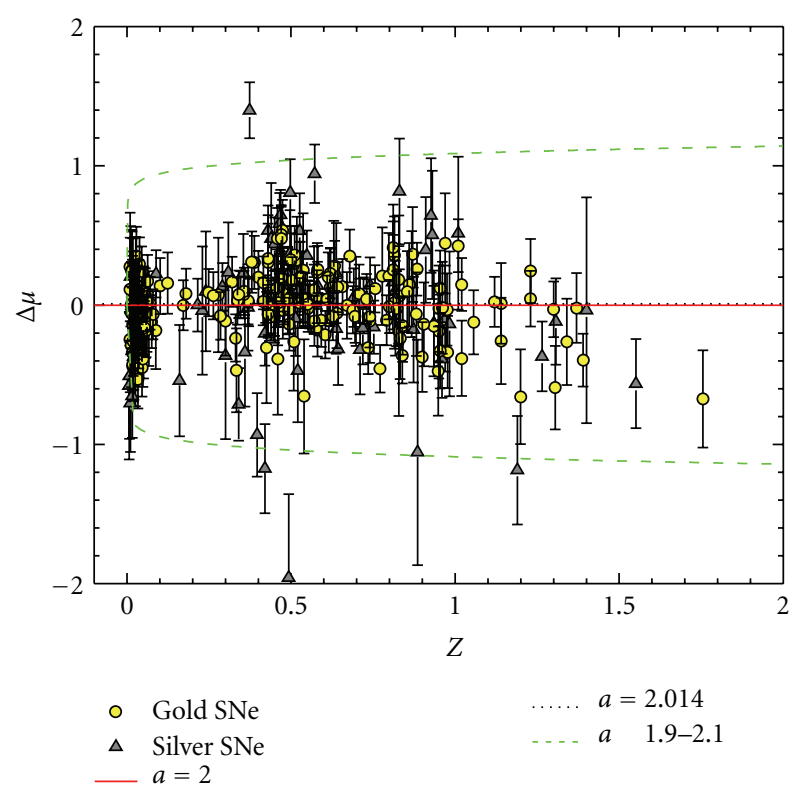

Figure 2: Data from type Ia SNe "gold-silver sets" [48] are fitted with (43) and shown as residuals $\Delta \mu$. The baseline is represented by our fit for fixed $a=2$ (solid red curve). The meaning of the other curves and parameters is the same as in Figure 1.

such as the one described in (44), is not easily integrated, so that the low-z behavior is usually studied by expanding it in Taylor series around $z=0$. This procedure yields the wellknown low-z expansion [15]

$$
d_{L}=\frac{c}{\mathbf{H}_{0}}\left[z+\frac{1}{2}\left(1-\mathbf{q}_{0}\right) z^{2}+\mathcal{O}\left(z^{3}\right)\right]
$$

The first-order term corresponds to the original Hubble's law, $v_{r} \simeq c z \simeq \mathbf{H}_{0} d_{L}$, where $v_{r}$ is the recessional velocity of the galaxy, following Hubble's original explanation based on a pure Doppler effect. The equivalent expansion for the distance modulus is

$$
\begin{aligned}
\mu(z)= & 25-5 \log _{10} \mathbf{H}_{0}+5 \log _{10}(c z) \\
& +1.086\left(1-\mathbf{q}_{0}\right) z+\mathcal{O}\left(z^{2}\right),
\end{aligned}
$$

where $\mathbf{H}_{0}$ is measured as usual in $\mathrm{km} \mathrm{s}^{-1} \mathrm{Mpc}^{-1}$ while $c=$ $299792.458 \mathrm{~km} \mathrm{~s}^{-1}$. These expressions need to be corrected, due to our new luminosity distance $d_{L}=\mathbf{R}_{0} \mathbf{r}$ from (22), as opposed to the standard cosmology expression $d_{L}=$ $(1+z) \mathbf{R}_{0} \mathbf{r}$. We also need to include the reference distance $d_{\mathrm{rs}}=\mathbf{R}_{0} \mathbf{r}_{\mathrm{rs}}=\mathbf{R}_{0}\left(2 \delta_{0} /\left(1-\delta_{0}^{2}\right)\right)$ at which we start observing the redshift.

Since our expression for $d_{L}$ in (22) is an explicit function of $z$, we can directly expand this function and obtain the following result (We note that if we were to correct the standard expansion formula, as given in [15] or [16], we would obtain $d_{L}=\left(c / \mathbf{H}_{\mathrm{rs}}\right)\left[z-(1 / 2)\left(1+\mathbf{q}_{\mathrm{rs}}\right) z^{2}+\mathcal{O}\left(z^{3}\right)\right]=$ $\mathbf{R}_{0}\left[\left(1 /\left|\delta_{0}\right|\right) z-\left(1 / 2\left|\delta_{0}\right|^{3}\right) z^{2}+\mathcal{O}\left(z^{3}\right)\right]$. This is the same result we would get by expanding our luminosity distance for a negative value $\delta_{0}<0$. This is because standard cosmology does not include the distance $d_{\mathrm{rs}}$ in the derivation, and this assumption is equivalent to considering the current value of $\delta$ as negative.):

$$
d_{L}=\mathbf{R}_{0}\left[\frac{2 \delta_{0}}{1-\delta_{0}^{2}}+\frac{1+\delta_{0}^{2}}{\delta_{0}\left(1-\delta_{0}^{2}\right)} z-\frac{1}{2 \delta_{0}^{3}} z^{2}+\mathcal{O}\left(z^{3}\right)\right],
$$

from which we could derive an expansion for the distance modulus $\mu(z)$. The problem with this type of expression is that it has a very limited range of validity in our revised cosmology, due to the small value of $\delta_{0} \simeq 3.83 \times 10^{-5}$ (or due to the large value of $\mathbf{q}_{0}=1 / \delta_{0}^{2}-1 \simeq 6.82 \times 10^{8}$ ). It is easy to check that, in our new expression for $d_{L}$ in the last equation, we could neglect the $z^{2}$ and higher-order terms only for $z \lesssim 10^{-9}$, which is a range of no practical interest. Therefore, a low- $\mathrm{z}$ analysis similar to the one of standard cosmology is actually not feasible in our new approach.

However, due to the low value of $\delta_{0} \simeq 3.83 \times 10^{-5}$, we can expand (22) in powers of $\delta_{0}$ around $\delta_{0}=0$ (or just consider (22) for $\delta_{0} \rightarrow 0$ ). With a Taylor expansion, we obtain

$$
\begin{aligned}
d_{L} & =\mathbf{R}_{0}\left[\sqrt{z^{2}+2 z}+(1+z) \delta_{0}+\mathcal{O}\left(\delta_{0}^{2}\right)\right], \\
\mu(z)= & 2.5(2+a) \log _{10} \\
& \times\left\{\frac{1-\delta_{0}^{2}}{2 \delta_{0}}\left[\sqrt{z^{2}+2 z}+(1+z) \delta_{0}+\mathcal{O}\left(\delta_{0}^{2}\right)\right]\right\} .
\end{aligned}
$$

The leading terms at low redshift of the expressions in the previous equation are the following (neglecting the $1-\delta_{0}^{2}$ factor in the second line, due to the small value of $\delta_{0}$ ):

$$
\begin{gathered}
d_{L} \simeq \mathbf{R}_{0} \sqrt{2 z}, \\
\mu(z) \simeq 2.5(2+a) \log _{10}\left(\frac{\sqrt{2 z}}{2 \delta_{0}}\right) .
\end{gathered}
$$

This equation can be considered our "low-z" expression, and we can check its validity by using the second line to fit the "gold" SNe data of [48] in the low-z regime. For this purpose, we selected the "gold" data with $z \lesssim 0.1$, with the same "double-correction procedure" mentioned above, and we used the last equation as a fitting formula. The results obtained are the following:

$$
\begin{gathered}
a=2.041 \pm 0.084, \\
\delta_{0}=(4.159 \pm 0.693) \times 10^{-5},
\end{gathered}
$$

$\left(\chi^{2}=0.0430 ; R^{2}=0.975\right)$ leaving $a$ variable. If we constrain $a$ to our preferred value, we obtain instead

$$
\begin{gathered}
a=2, \\
\delta_{0}=(3.836 \pm 0.023) \times 10^{-5},
\end{gathered}
$$

which is also a good-quality fit of the data $\left(\chi^{2}=0.0425 ; R^{2}=\right.$ 0.975). These results are shown in Figure 3, where the fit with the parameters of (54) is shown as a blue continuous curve, 


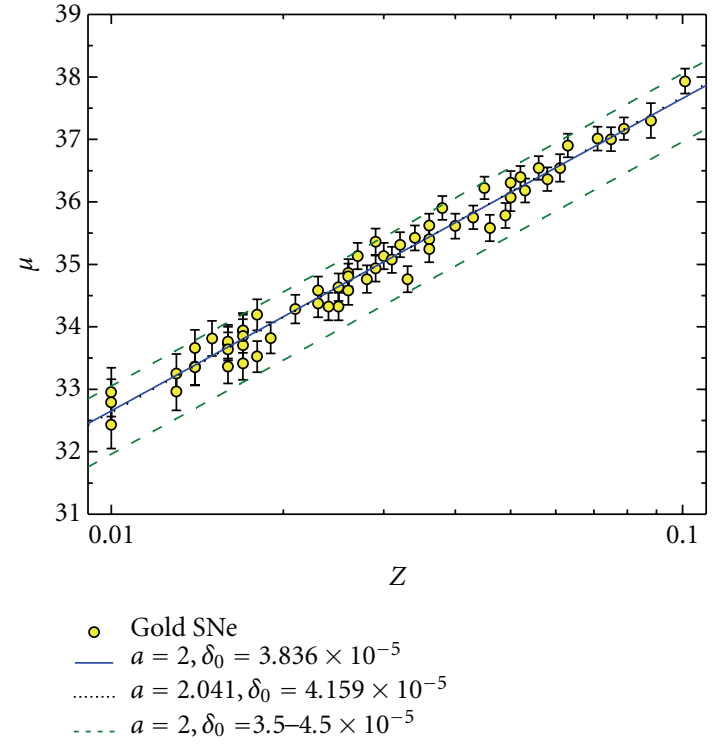

FIgURe 3: Data from type Ia SNe "gold set" [48] are fitted with (52), our low-z approximation. Our fits show a remarkably good quality $\left(\chi^{2}=0.043 ; R^{2}=0.975\right)$ for both a variable $a$ (black dotted curve) and for fixed $a=2$ (solid blue curve). In this last case the best fit parameter for delta is $\delta_{0}=3.836 \times 10^{-5}$. We also show (dashed green curves) the range of our fitting expressions for $\delta_{0}=3.5-$ $4.5 \times 10^{-5}$.

while the black-dotted line illustrates the fit with variable a. As in the previous figures, these two curves are virtually equivalent, confirming our hypothesis of an integer value for a.

In this figure, we also show (dashed green curves) our low-z fitting curve of (52) for $a=2$ and for the range $\delta_{0}=3.5-4.5 \times 10^{-5}$, just to illustrate how sensitive our fitting formula is to the value of $\delta_{0}$. Considering also the previous figures, we can see that the value of the parameter a determines the slope (or the shape of the curves in a logarithmic plot), while the value of $\delta_{0}$ basically determines the normalization of the curves, as it will also be shown in the following paragraphs.

Comparing these expressions with the standard cosmology ones in (48)-(49), it seems at first that our results do not yield the standard Hubble's law, $v_{r} \simeq c z \simeq \mathbf{H}_{0} d_{L}$, but as already remarked before, this law was introduced by Hubble following the original interpretation of the redshift as a pure (relativistic) Doppler shift. This interpretation was later generalized into the cosmological expansion, but this view is not shared by our model, and therefore we do not need to recover the original Hubble's law in our approach.

On the contrary, we can rewrite our "low-z" expression for the distance moduli in (52), with $a=2$, as

$$
\mu(z) \simeq 10 \log _{10}\left(\frac{\sqrt{2 z}}{2 \delta_{0}}\right)=5 \log _{10}\left(\frac{z}{2 \delta_{0}^{2}}\right)
$$

so that we have a perfect correspondence between our expression for $\mu(z)$ in the last equation and the classical one from (49), which can also be rewritten as

$$
\mu(z) \simeq 25+5 \log _{10}\left[\frac{c z}{\mathbf{H}_{0}}\right]=5 \log _{10}\left[10^{5} \frac{c z}{\mathbf{H}_{0}}\right],
$$

neglecting higher-order terms in $z$. Since both expressions (55) and (56) fit the experimental data and the standard expression can be used to measure the Hubble constant $\mathbf{H}_{0}$ (with $\mathbf{H}_{0} \simeq H_{0}$, see the discussion related to (30)) comparing them together and using the value of $\delta_{0}$ from (54), we obtain

$$
\mathbf{H}_{0} \simeq H_{0} \simeq 2 \times 10^{5} c \delta_{0}^{2}=88.2 \mathrm{~km} \mathrm{~s}^{-1} \mathrm{Mpc}^{-1} .
$$

This value is very close to the Hubble constant we obtained in Section 2.2 (see (9)) and that we used for our calibration of the "gold-silver" data. This result shows that our calibration procedure of the SNe data was essentially correct and links directly the Hubble constant to the fundamental parameter $\delta_{0}$, as shown in the last equation. (We also tried fitting the original low-z "gold" data, based on the standard value of $H_{0}=73 \mathrm{~km} \mathrm{~s}^{-1} \mathrm{Mpc}^{-1}$, proposed by Riess et al. in [12]. This resulted in a slightly different value of $\delta_{0} \simeq 3.461 \times 10^{-5}$, which is consistent with the Hubble constant used, in view of (57).)

In addition, (57) can be combined with (9) to give a direct relation between $\delta_{0}$ and $\gamma_{0}$,

$$
\gamma_{0} \simeq 1.296 \times 10^{-19} \delta_{0}^{2} \mathrm{~cm}^{-1},
$$

where the numerical factor in the previous equation is a consequence of the different units used to measure the Hubble constant. Using the value of $\delta_{0}$ from (54) or (47) into the last equation, we obtain $\gamma_{0} \simeq 1.9 \times 10^{-28} \mathrm{~cm}^{-1}$, as introduced in (41) and based on the discussion of the Pioneer anomaly. (We also performed a fit of the gold supernova data using (51), the "low- $\delta$ " expansion formula. Setting $a=2$, we obtained in this case $\delta_{0}=3.868 \times 10^{-5}$, which placed in (57) and (58) yields, respectively, $H_{0}=89.7 \mathrm{~km} \mathrm{~s}^{-1} \mathrm{Mpc}^{-1}$ and $\gamma_{0}=1.94 \times 10^{-28} \mathrm{~cm}^{-1}$, the exact values on which we base our calibration of supernova data. In this sense, our "low$\delta$ " expansion formula (51) should probably take the place of the "low-z" expression (52) as being more relevant to our analysis.)

Finally, in Figure 4, we reproduce the same data and fitting curves as in the previous figures, but in the form of a standard linear Hubble plot, to show that our approach can yield also these type of graphs, with the same quality of those of standard cosmology.

In particular, we see in this figure that our normal fit (in red, upper-solid curve) following (43) and the "low-z" fit (in blue, lower-solid curve) following (52) for a fixed $a=2$ are almost equivalent at low redshift as expected, but they become consistently different for $z \gtrsim 0.1$. Since our normal fit, based on $\delta_{0}=3.827 \times 10^{-5}$, can describe in a better way all the supernova data in our gold set, we will prefer this value over the low-z evaluation of (54). We will therefore use $\delta_{0} \simeq 3.83 \times 10^{-5}$ in the summary of the fundamental parameters presented in the following section. 


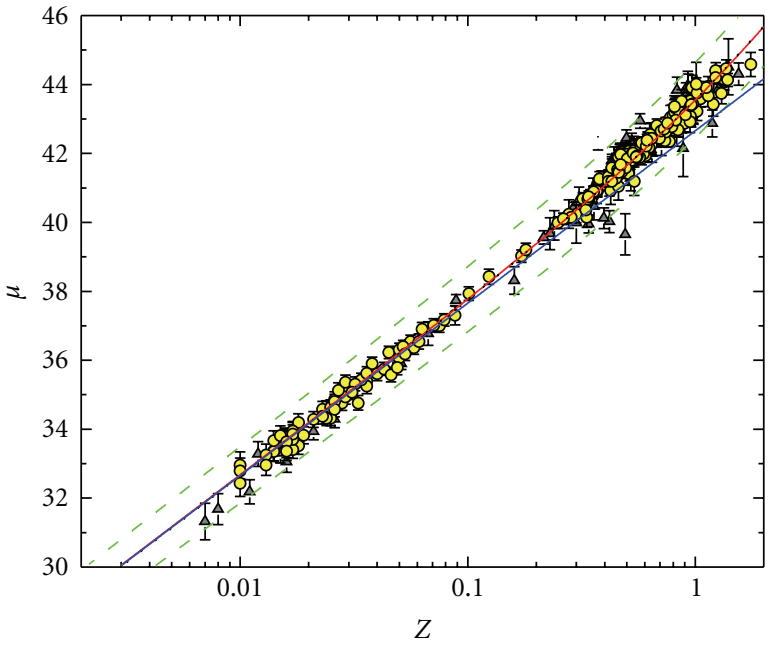

- Gold SNe

$\Delta \quad$ Silver $\mathrm{SNe}$ $a=2, \delta_{0}=3.827 \times 10^{-5}$, normal fit

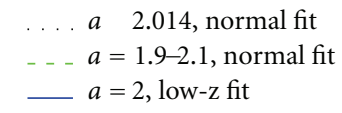

FIGURE 4: Data from type Ia SNe "gold-silver sets" [48] are fitted with (43) —normal fit (red, upper-solid curve for fixed $a=2$; black dotted for variable $a$ ) and (52)-low-z fit (blue, lower-solid curve). Also shown by the green-dashed curves is the range $a=1.9-2.1$ (normal fit).

3.3. The Other Cosmological Parameters. Using the results from the previous sections we are finally able to report our best estimates of the parameters which enter our kinematical conformal cosmology. These parameters are shown in Table 1, where all the quantities either refer to their current value (subscript zero) or to the values at the location where the redshift starts being observed (subscript rs). We used for all these estimates the value of $\delta_{0}$ from (47).

As it was previously mentioned, it is beyond the scope of this paper to perform a full revision of the "cosmological distance ladder," in view of the changes proposed by our new approach. However, we want to show here the difference between our luminosity distance estimates and the standard results, for different redshift values. Table 2 illustrates the results of this computation for different values of the redshift parameter: in the second column, we use our new definition of luminosity distance following (18), while in the third column, we employ the standard cosmology formula in (44) with $\Omega_{M}=0.27, \Omega_{\Lambda}=0.73, H_{0}=89.7 \mathrm{~km} \mathrm{~s}^{-1} \mathrm{Mpc}^{-1}$, to bring it in line with our preferred value of the Hubble constant.

As we can see from the table, there is a quite large difference between the estimates in these two columns, a difference of about three orders of magnitude just at low redshift, for $z \sim 0.01-0.1$. This is mainly due to our hypothesis of a change in the "absolute" luminosity of standard candles in (21), resulting in dramatically smaller revised distances.

However, if we were to redefine our new luminosity distance as in (23), that is, considering as in standard cosmology an invariable absolute luminosity $L_{0}$ of the source and using corrections due to an expansion of the Universe similar to standard cosmology, we would obtain

$$
\begin{aligned}
d_{L} & =\sqrt{\frac{L_{0}}{4 \pi l}}=\sqrt{(1+z) / f(1+z)} \mathbf{R}_{0} \mathbf{r} \\
& =\mathbf{R}_{0} \frac{\left[\delta_{0}(1+z)+\sqrt{(1+z)^{2}-\left(1-\delta_{0}^{2}\right)}\right]^{2}}{2 \delta_{0}\left(1-\delta_{0}^{2}\right)},
\end{aligned}
$$

where we used our explicit form of the function $f(1+z)$ in (42) with $a=2$.

In the fourth column of Table 2, we used the previous equation to compute the distances, and we notice that these values are close to those of the standard cosmology for both $H_{0}=89.7 \mathrm{~km} \mathrm{~s}^{-1} \mathrm{Mpc}^{-1}$ (third column) or for the more standard value $H_{0}=73 \mathrm{~km} \mathrm{~s}^{-1} \mathrm{Mpc}^{-1}$ (values in the last column of the table). This is of course expected, since the distance in (59) would also fit the supernova data, if we were to assume an expansion equivalent to that of standard cosmology. In other words, (59) is our equivalent of the "standard" luminosity distance and yields to virtually the same distance estimates as in standard cosmology. However, in our interpretation, (18) is to be considered the correct distance, since it includes the intrinsic dimming of the source.

We wanted to introduce also this "standard-equivalent" luminosity distance in (59), in order to make a comment on the so-called "Tolman surface brightness test," which is usually employed in cosmology to distinguish between standard expansion theories and alternative models of redshift, such as the "tired light" explanation (see discussion in Section 1.7 of [16] and recent experimental results in [54]). This test is based on the ratio between the angular diameter distance $d_{A}$ and the luminosity distance $d_{L}$. Using standard cosmology distances from (16) and (25), this ratio is simply $d_{A} / d_{L}=(1+z)^{-2}$, so that the surface brightness $B$ of a luminous object (defined as the apparent luminosity per solid angle- $-l / \Omega)$ will result in $B \equiv l / \Omega=(\mathcal{L} / 4 \pi)\left(d_{A} / d_{L}\right)^{2}=$ $(\mathcal{L} / 4 \pi)\left(1 /(1+z)^{4}\right)$, where $\mathcal{L}$ is the intrinsic absolute luminosity per unit of proper area of the source (see [16] for details). As shown in the above equation, this quantity should scale like $(1+z)^{-4}$, and this prediction is recovered (within certain limits, due to the evolution of the galactic light sources) in experimental studies [54]. Tired light theories would require $B$ to scale as $(1+z)^{-1}$ and are essentially ruled out by these experimental evidences.

In our model, the ratio $d_{A} / d_{L}$, constructed using (18) and (26), does not seem to scale as required by the Tolman brightness test, that is, $d_{A} / d_{L}=(1+z)^{-2}$, but rather as $(1+z)^{-1}$, yielding a surface brightness scaling as $(1+z)^{-2}$. However, experimental tests of this effect, such as the one reported in [54], are based on a standard approach using invariable luminosities and invariable diameters of the light sources being studied. Following the discussion in the previous paragraphs, this amounts to using our "standardequivalent" luminosity distance in (59), instead of the one in (18). We can see from the fourth column in our Table 2 that distances computed with this "standard-equivalent" 
TABle 1: The fundamental parameters of our kinematical conformal cosmology are shown here, as derived from the astrophysical data analyzed in the current section.

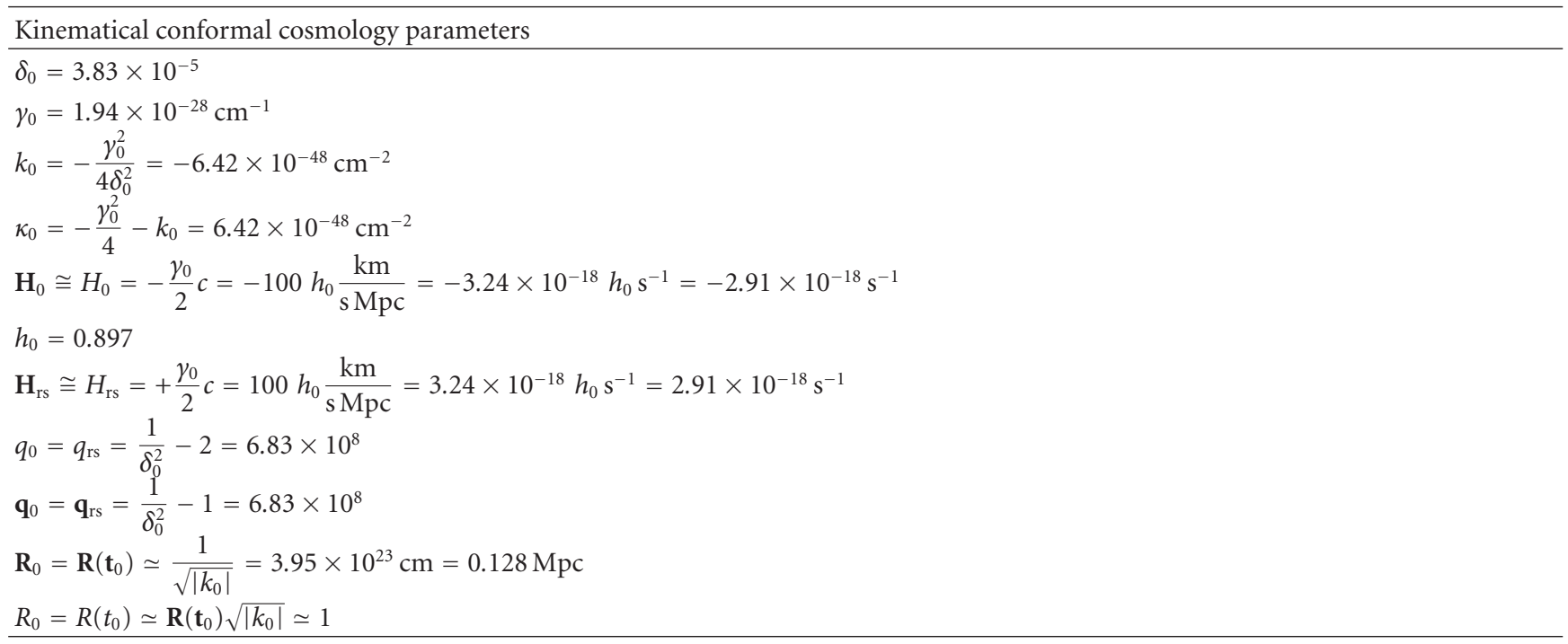

TABLE 2: Comparison between luminosity distances in our model and in standard cosmology, for different values of the redshift parameter.

\begin{tabular}{lcccc}
\hline$z$ & $d_{L}(\mathrm{Mpc})(18)$ & $d_{L}(\mathrm{Mpc})(44)$ & $d_{L}(\mathrm{Mpc})(59)$ & $\begin{array}{c}d_{L}(\mathrm{Mpc})(44) \\
H_{0}=73(\mathrm{~km} / \mathrm{s} \mathrm{Mpc})\end{array}$ \\
\hline 0.001 & $H_{0}=89.7(\mathrm{~km} / \mathrm{s} \mathrm{Mpc})$ & $H_{0}=89.7(\mathrm{~km} / \mathrm{s} \mathrm{Mpc})$ & $H_{0}=89.7(\mathrm{~km} / \mathrm{s} \mathrm{Mpc})$ & 4.11 \\
0.01 & $5.73 \times 10^{-3}$ & 3.35 & 3.35 & 41.4 \\
0.1 & $1.81 \times 10^{-2}$ & 33.7 & 33.6 & 442 \\
1 & $5.86 \times 10^{-2}$ & 360 & $5.02 \times 10^{3}$ & $6.45 \times 10^{3}$ \\
10 & 0.221 & $5.25 \times 10^{3}$ & $2.01 \times 10^{5}$ & $1.03 \times 10^{5}$ \\
100 & 1.40 & $8.41 \times 10^{4}$ & $1.71 \times 10^{7}$ & $1.27 \times 10^{6}$ \\
1000 & 12.9 & $1.04 \times 10^{6}$ & $1.68 \times 10^{9}$ & $1.37 \times 10^{7}$ \\
\hline
\end{tabular}

expression are similar to those calculated by standard cosmology. Therefore, we infer that current tests of the Tolman effect would not be in disagreement with our model. We will leave to future work a more detailed analysis of this effect.

In any case, if our approach is correct and we use our new luminosity distance in (18), other distance estimates in the cosmological ladder might also need to be revised. We mentioned in Section 2.3 that several other distances need to be changed and that, for example, our new angular diameter distance would imply larger distances than previously thought; therefore, the overall reduction in distance estimates might be less dramatic than the one illustrated in Table 2, comparing just the second and third columns.

This is related to the final point we want to address in this section, that is, the apparent discrepancy between our estimate of the parameter $\gamma_{0}=1.94 \times 10^{-28} \mathrm{~cm}^{-1}$ and the value proposed by Mannheim, $\gamma_{\text {Mannheim }}=3.06 \times$ $10^{-30} \mathrm{~cm}^{-1}$ [8]. We recall that this value was obtained by Mannheim using a sample of eleven galaxies, where the rotational motion data were fitted by the conformal gravity theory over a range of radial distances of a few kiloparsec, from the center of each galaxy. The non-Keplerian effects are manifest beyond the peak value at $r_{\text {peak }}=2.2 r_{0}$, with $r_{0}$ ranging from $0.48 \mathrm{kpc}$ to $4.48 \mathrm{kpc}$, for the sample of eleven galaxies. The average $r_{\text {peak }}$ is about $4.27 \mathrm{kpc}$; therefore, the measured $\gamma_{0}$ was obtained by fitting the original MannheimKazanas potential over radial distances $r \gtrsim r_{\text {peak-ave }}=$ $4.27 \mathrm{kpc}$ from the reference point of observation (the center of each galaxy). The global redshift of each galaxy was already subtracted from the rotational data; therefore, the measured $\gamma_{0}$ refers to the intrinsic scale of the galaxies being considered (kiloparsec scale) and should be in line with the value we propose.

However, Mannheim's analysis was based on standard cosmology estimates of the distances to all these galaxies. We have seen above that our new interpretation of the luminosity distance implies rather smaller distances than those previously estimated. The ratio between the two estimates of the gamma parameter

$$
\gamma_{0} / \gamma_{\text {Mannheim }}=63.4
$$

could be explained in terms of a similar ratio between distance estimates in the standard theory versus our new approach. This is due to the fact that, as mentioned in our paper I, galactic rotational curve was fitted by Mannheim using a potential proportional to the quantity $\gamma r$, so that 
the overestimation of the distances $r$ would result in an underestimation of the $\gamma$ parameter. We will also leave to future work a more detailed analysis of this issue.

\section{Consequences of the Model}

To conclude the analysis of our kinematical conformal cosmology, we want to summarize in this section the scaling properties of all the dimensionful quantities and link these results to the more general problem of the time variation of physical constants. This is followed by a similar analysis of the dimensionless parameters and constants, which might establish a direct connection between our parameter $\delta$ and the fine-structure constant $\alpha_{\mathrm{em}}$ of the electromagnetic theory.

4.1. Scaling Properties of Dimensionful Quantities. In our approach to cosmology, all physical quantities with dimensions (of length, time, mass, etc.) are affected by the general "scaling" properties detailed by (19), with the function $f(1+$ $z$ ) defined as in (42), where we use $a=2$ as our preferred value for the inverse-power law generalization. In particular, using the relations in Table I of our first paper [7], we can express all these scaling factors as a function of $z$ and $\delta_{0}$, or as a function of the "cosmological time" $\delta$ and its current value $\delta_{0}$, since we have

$$
\begin{aligned}
1+z & =\sqrt{\frac{1-\delta_{0}^{2}}{1-\delta^{2}}}, \\
f(1+z) & =\frac{4 \delta_{0}^{2}(1+z)}{\left[\delta_{0}(1+z)+\sqrt{(1+z)^{2}-\left(1-\delta_{0}^{2}\right)}\right]^{2}} \\
& =\frac{4 \delta_{0}^{2}}{\left[\delta_{0}+|\delta|\right]^{2}} \sqrt{\frac{1-\delta^{2}}{1-\delta_{0}^{2}}} .
\end{aligned}
$$

These equations imply that when we observe the Universe at a certain fixed value of the cosmological time (e.g., the current value $\delta_{0}$ ) all dimensionful quantities and constants appear to scale with the redshift parameter $z$ (or with $\delta$ varying from -1 to +1$)$. This will also affect the fundamental constants of physics (with the exception of the speed of light, as already discussed in our paper I).

For example, Planck's constant, whose current value is $h_{0}=6.626 \times 10^{-27} \mathrm{ergs}$, would be perceived as scaling like the product of a mass and a length, resulting in the following:

$$
\begin{aligned}
h & =h_{0}(1+z) f(1+z) \\
& =h_{0} \frac{4 \delta_{0}^{2}(1+z)^{2}}{\left[\delta_{0}(1+z)+\sqrt{(1+z)^{2}-\left(1-\delta_{0}^{2}\right)}\right]^{2}}=h_{0} \frac{4 \delta_{0}^{2}}{\left[\delta_{0}+|\delta|\right]^{2}} .
\end{aligned}
$$

This equation implies that if we could observe an experiment designed to measure $h$ and performed at a location characterized by redshift $z$, the outcome of this experiment would be Planck's constant as given by (62) and not our current standard value $h_{0}$.

Another example of these scaling properties is related to the elementary charge $e_{0}=4.803 \times 10^{-10}$ stat $C$ (current value in cgs units). Whenever we observe the Universe at a fixed cosmological time, it is natural to assume that all dimensionless quantities should also be considered fixed at the particular values they have at that cosmological time. In particular, the fine-structure constant of electromagnetism is defined as the (dimensionless) quantity $\alpha_{\mathrm{em}}=e^{2} / \hbar c$ (with $\hbar=h / 2 \pi)$ and has a current value of $\alpha_{\mathrm{em}_{0}}=7.297 \times$ $10^{-3}$. This definition of $\alpha_{\mathrm{em}}$ implies that the square of the elementary charge, $e^{2}$, should scale as Planck's constant (since $c$ does not scale and $\alpha_{\mathrm{em}}$ is assumed to be a fixed dimensionless quantity). Therefore, the elementary charge $e$ should scale as

$$
\begin{aligned}
e & =e_{0} \sqrt{(1+z) f(1+z)} \\
& =e_{0} \frac{2 \delta_{0}(1+z)}{\left[\delta_{0}(1+z)+\sqrt{(1+z)^{2}-\left(1-\delta_{0}^{2}\right)}\right]}=e_{0} \frac{2 \delta_{0}}{\left[\delta_{0}+|\delta|\right]},
\end{aligned}
$$

and similar scaling properties can be obtained for other physical constants of interest.

4.2. Dimensionless versus Dimensionful Quantities. Using the equations outlined in the previous sections we can compute the scaling properties of all quantities with physical dimensions, as seen from an observer at the current cosmological time $\delta_{0}$. However, we argue that the origin of the variability of all these quantities should be found in the values of dimensionless parameters and constants. In fact, we have shown above how the scaling properties can be described in terms of a variable dimensionless $\delta$ parameter.

An alternative but equivalent way of describing these effects is to connect them to a variable fine-structure constant $\alpha_{\mathrm{em}}$, that is, to assume that a dimensionless quantity such as $\alpha_{\text {em }}$ will vary over cosmological times, following changes of the $\delta$ parameter. We recall that the wavelength of emitted radiation, in a first-order approximation, can be related to $\alpha_{\mathrm{em}}$ as follows:

$$
\lambda \sim \frac{h}{m c \alpha_{\mathrm{em}}^{2}},
$$

since the Rydberg constant for infinite nuclear mass is $R_{\infty}=m_{e} c \alpha_{\mathrm{em}}^{2} / 2 h$, and the wavelength of emitted radiation is inversely proportional to $R_{\infty}$, as stated by the simple Balmer's formula. In (64), $m$ can be considered to be the electron mass $m_{e}$, or the reduced mass of the atomic system emitting the radiation.

If we assume that the cosmological redshift or blueshift is due to the intrinsic changes of a dimensionless quantity such as $\alpha_{\mathrm{em}}$, we are induced to consider the dimensionful constants such as $h$ and $m$ as fixed, since their changes are only apparent when we observe them at a fixed value of 


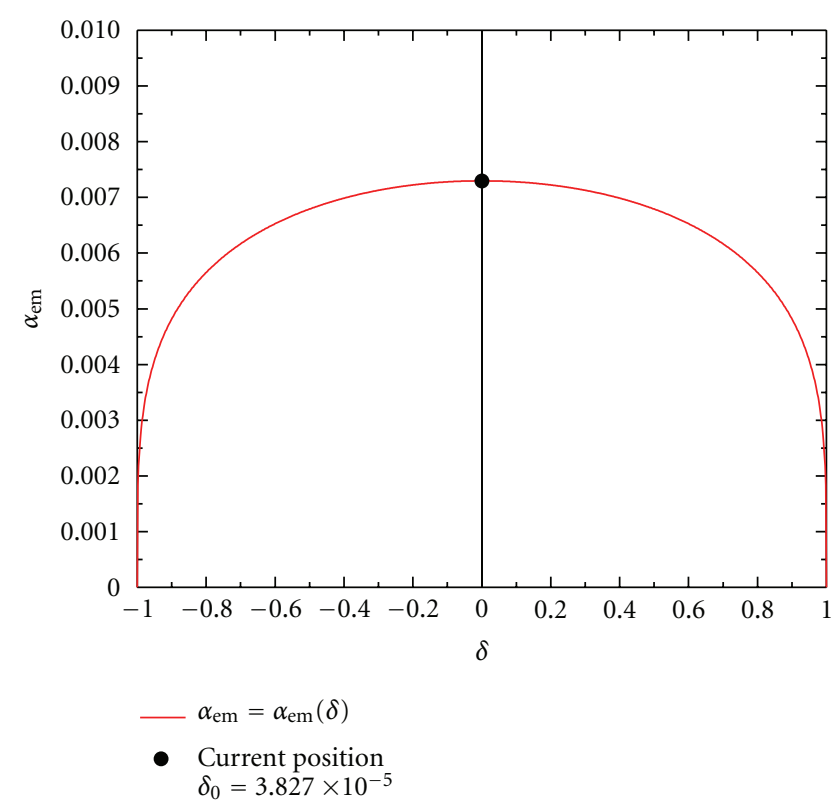

Figure 5: The dependence of $\alpha_{\mathrm{em}}$ on our parameter $\delta$ is shown, given the current values of these two quantities, $\alpha_{\mathrm{em}_{0}}=7.297 \times 10^{-3}$ and $\delta_{0}=3.827 \times 10^{-5}$ (red solid curve). Our current "position" in the plot is indicated by the black dot in the figure.

the dimensionless $\delta$ parameter. In this line of reasoning, we can rewrite the first expression in (61) as

$$
1+z=\sqrt{\frac{1-\delta_{0}^{2}}{1-\delta^{2}}}=\frac{\lambda}{\lambda_{0}}=\frac{\alpha_{\mathrm{em}}^{2}}{\alpha_{\mathrm{em}}^{2}},
$$

having used (64) with the dimensionful quantities considered fixed.

We therefore obtain how the fine-structure constant changes with $z$ or $\delta$,

$$
\alpha_{\mathrm{em}}=\frac{\alpha_{\mathrm{em}_{0}}}{\sqrt{1+z}}=\alpha_{\mathrm{em}_{0}} \sqrt[4]{\frac{1-\delta^{2}}{1-\delta_{0}^{2}}},
$$

which is illustrated in Figure 5, where the dependence of $\alpha_{\mathrm{em}}$ on our parameter $\delta$ is shown, given the current values of these two quantities, $\alpha_{\mathrm{em}_{0}}=7.297 \times 10^{-3}$ and $\delta_{0}=3.827 \times$ $10^{-5}$. The red continuous curve in the figure shows how the fine-structure constant is zero for $\delta=-1$; it is increasing to its maximum value $\alpha_{\mathrm{em}}^{\max }=\alpha_{\mathrm{em}} \sqrt[4]{1 /\left(1-\delta_{0}^{2}\right)}$ for $\delta=0$ and then decreases to zero again for $\delta=+1$. Our current "position" in the plot is indicated by the black dot in the figure. Given the very small value of $\delta_{0}$, the current value $\alpha_{\mathrm{em}}=7.297 \times 10^{-3}$ is basically the same as the maximum, indicating that electromagnetic interactions are currently at their strongest level.

Therefore, if our model is correct, we can consider dimensionless quantities such as $\delta$ and $\alpha_{\mathrm{em}}$ as the fundamental physical parameters directly connected to the evolution of the Universe. Their values are changing with time, or they can directly represent the cosmological time. The values of the dimensionful constants and parameters are merely a consequence of the current values of the dimensionless quantities, which determine the standard units with which all quantities with physical dimensions are measured.

Einstein was one of the first scientists to advocate for the importance of dimensionless quantities over dimensionful ones, as shown in a private correspondence with a former student of his (see discussion in Chapter 3 of Barrow's book [55]). Einstein thought that constants with physical dimensions are merely a product of the units of measure being used and as such they do not possess a deep theoretical meaning. On the contrary, dimensionless quantities constructed with standard dimensionful constants (such as $\alpha_{\mathrm{em}}=e^{2} / \hbar c$ ) are considered by Einstein to be the only significant numbers in physics, whose value should be possible to explain in terms of fundamental mathematical constants such as $\pi$ or $e$.

Our approach, described in this section, follows Einstein's consideration of dimensionless quantities as being the most fundamental ones, but we have shown above that their values are probably also changing with the universal time. Therefore, there is no need to explain a particular current value of $\alpha_{\mathrm{em}}$ or $\delta$, but it is sufficient to describe the evolution of these parameters.

We also remark that our analysis of the time variability of fundamental constants is different from the standard approach to this subject (for reviews, see [55-57]). For example, in recent claims of a (very small) time variability of the fine-structure constant, as seen in interstellar absorption spectra [58], the cosmological redshift is obviously factored out from the effect being studied, thus resulting in a very small variation of $\alpha_{e m}$ over cosmological scales. On the contrary, in our approach, the cosmological redshift is potentially explained in terms of a (large) variation of the finestructure constant, as seen in Figure 5, therefore resulting in a totally different phenomenology.

It is beyond the scope of this work to extend this analysis to all other fundamental constants in nature (dimensionless or not). We simply point out that if our hypothesis on the variability of physical constant is correct, this would call for a revision of the theory of fundamental interactions, such as quantum electrodynamics or others, in view of variable coupling constants and interaction strengths.

\subsection{Astrophysical Observations and Kinematical Conformal} Cosmology. We have seen that in our approach to cosmology experiments and observations based on atomic properties and electromagnetic phenomena are affected by our new interpretation. These possible changes in electromagnetic physics (and perhaps also in strong/weak interactions) over cosmological times will affect our observations and perspective of the gravitational motion.

The description of the Universe can still be done with the standard Newton-Einstein paradigm, assuming invariable space-time units and measuring all quantities with these fixed units. Such is the case when we use "ranging" techniques, that is, we measure the time of flight of light signals to determine the positions of celestial bodies.

On the contrary, other astrophysical observations (such as those based on spectroscopy or similar) need to include the stretching of space-time and the change of related units. 
In this case we need conformal gravity as an enhancement of general relativity, to include this "stretching" of the units, and our kinematical approach might help to explain the shortcomings of the standard theory.

In order to test our model, a conclusive experiment would be an enhanced measurement of the "Pioneer anomaly" effect, possibly realized through a dedicated mission of a spaceship in the outer solar system, as it has already been proposed [33-35]. This seems to be the only practicable type of experiment in which an electromagnetic signal of wellknown wavelength can be transmitted unperturbed over a considerable temporal interval. In this way, it can be compared to a similar signal produced at a later time to check for an intrinsic wavelength shift, due to the conformal stretching of the space-time. The direct detection of such a wavelength/frequency shift of an electromagnetic signal with time would be a clear signature of conformal gravity acting according to our kinematical approach.

Further work will also need to be done in order to check if our model is consistent with the presence of the cosmic microwave background radiation, which is one of the experimental cornerstones of standard cosmology, and also improve our previous estimates of the age of the Universe [7], and of the other cosmological parameters, in order to validate our model.

Finally, we remark that a detailed analysis and comparison between standard $\Lambda$ Cold Dark Matter $(\Lambda \mathrm{CDM})$ cosmology and conformal gravity models has recently appeared [59]. In particular, Mannheim's conformal gravity (CG) and our kinematical conformal gravity (KCG), as described in this paper, were used to model combined data from type Ia Supernovae and Gamma-ray bursts, using a Bayesian analysis of the data. The outcome of this work was that "Contrary to the expectation, we show that the current data can be described by CG, KCG, and $\Lambda$ CDM equally well" (quoted from [59]). This result is particularly encouraging to further continue our studies of kinematical conformal cosmology also in the future.

\section{Conclusions}

We have introduced experimental evidence in support of our kinematical conformal cosmology and determined the values of its fundamental parameters. In particular, we have focused our analysis on reproducing the Hubble plots for type Ia supernovae, with the same level of accuracy obtained by standard cosmology.

To achieve this goal, we critically reconsidered all the standard distances commonly used in cosmology and added a new scaling property for mass (or energy) as a function of the redshift parameter. Our new expression for the distance modulus as a function of redshift can effectively fit the "goldsilver" SNe data with the required accuracy and also yield a current-time value for our fundamental parameter $\delta_{0}$, which is small and positive as expected.

Since type Ia supernovae or other astrophysical candles are distant cosmological objects, our second point of focus was to consider more local effects due to our kinematical conformal cosmology, which might be more suitable for the determination of the parameters. In particular, a local blueshift region was expected, given the estimated values of the quantities in our model, and is possibly evidenced by the recently discovered Pioneer anomaly.

We have seen how our model can account for this effect and can be used to estimate our second fundamental parameter $\gamma_{0}$, which together with $\delta_{0}$ will determine all the other quantities in our model. More precise evaluations of these parameters are certainly needed and should come from an extended analysis of the Pioneer data or through a dedicated future spacecraft mission, which has already been proposed.

We argued that a direct detection of a frequency/wavelength shift in electromagnetic radiation, traveling over distances comparable to the size of our solar system, could be explained only in terms of a conformal space-time stretching and would be the best evidence in support of our kinematical approach. If the Pioneer anomaly, or similar phenomena, will prove in the next few years to be positive indications of these effects, they might also signal a possible time variation of dimensionless fundamental quantities, such as the finestructure constant and our cosmological time $\delta$. This would constitute an important step towards a deeper understanding of the role and values of all the fundamental physical constants and also impact our current understanding of the fundamental interactions.

\section{Appendix}

\section{K-Corrections in Luminosity Measurements}

In this appendix, we will briefly review the theory of Kcorrections used in luminosity measurements of standard candles (especially type Ia SNe) and check if our new cosmology will require any changes in the definition of such corrections. We recall that a luminosity distance versus magnitude equation, such as (17) used in standard cosmology or our revised formula (43), usually relates the theoretical expression of $d_{L}$ to the "bolometric" apparent and absolute magnitudes of the light source, that is, considering radiation emitted over the whole wavelength spectrum.

On the contrary, CCD detectors or other photometric devices used in astronomy usually observe radiation within certain wavelength bands (such as those related to the $U, B$, $V, R$, and $I$ filters mentioned in Section 3.2), and this information needs to be converted into bolometric magnitudes before it can be used in the luminosity distance equations. "K-corrections" are introduced for this purpose. Moreover, due to the large redshifts of some of the SNe being observed, photometry in a highly redshifted filter (such as in the $R$ band) has sometimes to be compared to nearby photometry of a reference supernova in a different filter (such as $B, V$, or other), thus involving also conversions between different filters, in addition to the standard correction from one band to bolometric magnitudes.

The theory of K-corrections was originally introduced by Humason et al. [60] and later reviewed by Oke and Sandage [61] in 1968. It was later adapted to the modern case of type Ia SNe spectra in a series of papers (see, e.g., [62-64]). In this 
appendix, we will follow the notation used in these modern reviews.

The standard K-correction connects the apparent magnitude $m_{x}$ in some " $x$ " filter band of a light source at redshift $z$, to the distance modulus $\mu(z)$ according to the following equation [62]:

$$
\begin{aligned}
\mu(z)=m_{x}\left(z, t_{\mathrm{obs}}\right)-M_{x}\left(z=0, t_{\text {rest }}\right)-K_{x}\left(z, t_{\text {rest }}\right), \\
K_{x}\left(z, t_{\text {rest }}\right)=2.5 \log _{10}(1+z) \\
+2.5 \log _{10}\left\{\frac{\int F(\lambda) S_{x}(\lambda) d \lambda}{\int F[\lambda /(1+z)] S_{x}(\lambda) d \lambda}\right\},
\end{aligned}
$$

where $M_{x}$ is the absolute $x$ magnitude, and the appropriate correction $K_{x}$ is detailed in the second line of the equation, with $F(\lambda)$ being the spectral energy distribution "at the source" and $S_{x}(\lambda)$ the filter transmission. The time variables are connected by the standard time dilation equation $t_{\mathrm{obs}}=$ $t_{\text {rest }}(1+z)$, where $t_{\text {rest }}$ is the time in the supernova rest frame, and $t_{\mathrm{obs}}$ is the time in the observer frame. These times correspond, respectively, to our times $t_{z}$ and $t_{0}$ as discussed in Section 2.3, related in the same way, but with a different interpretation. We will omit the time dependence in the following since it is not essential for our discussion.

Following our revision of the luminosity distance, detailed in Section 2.3, we will recompute in our new notation the $K_{x}$ term and compare it to the standard expression in (A.1). Since $\mu(z)=m(z)-M(z=0)$, where $m$ and $M$ are bolometric magnitudes, the standard $\mathrm{K}$-correction is actually defined as

$$
\begin{aligned}
& K_{x}(z)= {\left[m_{x}(z)-M_{x}(z=0)\right]-[m(z)-M(z=0)] } \\
&=-2.5 \log _{10}\left[l_{x}(z) / l_{x}(z=0)\right] \\
&+2.5 \log _{10}\left[l_{\mathrm{bol}}(z) / l_{\mathrm{bol}}(z=0)\right] \\
&=-2.5 \log _{10}\left[\frac{\int\left(d L_{z} / d \lambda\right)\left(\lambda_{z}\right) S_{x}\left(\lambda_{z}\right) d \lambda_{z}}{4 \pi d_{L}^{2}}\right. \\
&\left.\times \frac{4 \pi d_{\mathrm{ref}}^{2}}{\int\left(d L_{0} / d \lambda\right)\left(\lambda_{0}\right) S_{x}\left(\lambda_{0}\right) d \lambda_{0}} \frac{L_{0}}{4 \pi d_{\mathrm{ref}}^{2}} \frac{4 \pi d_{L}^{2}}{L_{z}}\right] \\
&= 2.5 \log _{10}\left(\frac{L_{z}}{L_{0}}\right)+2.5 \log _{10}\left[\frac{\int F_{0}\left(\lambda_{0}\right) S_{x}\left(\lambda_{0}\right) d \lambda_{0}}{\int F_{z}\left(\lambda_{z}\right) S_{x}\left(\lambda_{z}\right) d \lambda_{z}}\right] \\
&+2.5 \log _{10}\left[\frac{f(1+z)}{(1+z)}\right] \int F_{0}\left(\lambda_{0}\right) S_{x}\left(\lambda_{0}\right) d \lambda_{0} \\
&+2.5 \log _{10}\left[\frac{\int F_{0}\left(\lambda_{0}\right) S_{x}\left(\lambda_{0}\right) d \lambda_{0}}{\int F_{0}\left[\lambda_{z} /(1+z)\right] S_{x}\left(\lambda_{z}\right) d \lambda_{z}}\right] \\
&= 2.5 \log _{10}\left[\frac{f(1+z)}{(1+z)}\right]+2.5 \log _{10}\left[\frac{(1+z)^{2}}{f(1+z)}\right] \\
& \hline
\end{aligned}
$$

$$
\begin{aligned}
= & 2.5 \log _{10}(1+z) \\
& +2.5 \log _{10}\left[\frac{\int F_{0}(\lambda) S_{x}(\lambda) d \lambda}{\int F_{0}[\lambda /(1+z)] S_{x}(\lambda) d \lambda}\right] .
\end{aligned}
$$

In this derivation, we used our new notation for all the functions and variables involved, including the connection between wavelengths $\lambda_{z}=\lambda_{0}(1+z)$, the spectral distribution "at the source" $F_{0}\left(\lambda_{0}\right) \equiv\left(d L_{0} / d \lambda\right)\left(\lambda_{0}\right)$, and the spectral distribution with the source placed at redshift $z$ and observed from the origin, $F_{z}\left(\lambda_{z}\right) \equiv\left(d L_{z} / d \lambda\right)\left(\lambda_{z}\right)$. We also used (21), (24) in the chain of derivation and renamed in the last line the wavelengths $\lambda_{z}, \lambda_{0}$ simply as $\lambda$, to obtain exactly the standard result of (A.1).

Therefore, the K-corrections computed according to the standard theory are unchanged in our kinematical conformal cosmology although, checking the derivation of (A.2) and comparing it to the same derivation of the standard theory, we observe that the final two terms originate in a slightly different way (see, e.g., how the $f(1+z)$ function cancels out in our case) and do not have the same meaning as those in the standard theory [61].

When two different filters are used in the observations, for example, the high-redshift photometry is observed with a " $y$ " filter and related to the nearby reference photometry in the " $x$ " filter, a new $K_{x y}$ correction is used, defined as [62]

$$
\begin{aligned}
\mu(z)= & m_{y}\left(z, t_{\text {obs }}\right)-M_{x}\left(z=0, t_{\text {rest }}\right)-K_{x y}\left(z, t_{\text {rest }}\right), \\
K_{x y}\left(z, t_{\text {rest }}\right)= & 2.5 \log _{10}(1+z) \\
& +2.5 \log _{10}\left\{\frac{\int F(\lambda) S_{x}(\lambda) d \lambda}{\int F[\lambda /(1+z)] S_{y}(\lambda) d \lambda}\right\} \\
& -2.5 \log _{10}\left\{\frac{\int Z(\lambda) S_{x}(\lambda) d \lambda}{\int Z(\lambda) S_{y}(\lambda) d \lambda}\right\} .
\end{aligned}
$$

The meaning of the terms and variables is similar to that of (A.1) and the main difference is the addition of a third term in the K-correction, which accounts for the differences in the zero points of the two filters $(Z(\lambda)$ is an idealized stellar spectral energy distribution at $z=0$ for which $U=B=V=$ $R=I=0$ in the photometric system being used [62]). The previous expression reduces to the standard K-term for the case when the two filters are the same and the third term added in (A.3) is a mere technical correction due to the different zero points used, thus unaffected by our new approach.

In conclusion, our new approach does not practically change the computation of $\mathrm{K}$-corrections as done in the standard theory; therefore, it was correct to use the experimental values of the distance moduli of the "gold-silver" type Ia SNe data [48] in our analysis in Section 3.2. 


\section{Acknowledgment}

The author wishes to thank the anonymous reviewers for their useful comments and suggestions which helped to improve the contents of this paper.

\section{References}

[1] H. Weyl, "Reine infinitesimalgeometrie," Mathematische Zeitschrift, vol. 2, no. 3-4, pp. 384-411, 1918.

[2] H. Weyl, "Gravitation und elekrizitaet," Koniglich Preussische Akademie der Wissenschaften, pp. 465-478, 1918.

[3] H. Weyl, "Eine neue erweiterung der relativitaetstheorie," Annalen der Physik, vol. 59, pp. 101-103, 1919.

[4] P. D. Mannheim and D. Kazanas, "Exact vacuum solution to conformal Weyl gravity and galactic rotation curves," Astrophysical Journal, vol. 342, p. 635, 1989.

[5] D. Kazanas and P. D. Mannheim, "General structure of the gravitational equations of motion in conformal weyl gravity," Astrophysical Journal, vol. 76, no. 2, pp. 431-453, 1991.

[6] P. D. Mannheim, "Alternatives to dark matter and dark energy," Progress in Particle and Nuclear Physics, vol. 56, no. 2, pp. 340-445, 2005.

[7] G. U. Varieschi, "A kinematical approach to conformal cosmology," General Relativity and Gravitation, vol. 42, no. 4, pp. 929-974, 2010.

[8] P. D. Mannheim, "Are galactic rotation curves really flat?" Astrophysical Journal, vol. 479, no. 2, pp. 659-664, 1997.

[9] K. R. Lang, Astrophysical Formulae: Vol. 1: Radiation, Gas Processes and High Energy Astrophysics. Vol. 2: Space, Time, Matter and Cosmology, Springer, Berlin, Germany, 1999.

[10] D. N. Spergel, R. Bean, O. Doré et al., "Three-year Wilkinson Microwave Anisotropy Probe (WMAP) observations: implications for cosmology," Astrophysical Journal, vol. 170, no. 2, pp. 377-408, 2007.

[11] W. L. Freedman, B. F. Madore, B. K. Gibson et al., "Final results from the Hubble Space Telescope key project to measure the Hubble constant," Astrophysical Journal, vol. 553, no. 1, pp. 4772, 2001.

[12] A. G. Riess, W. Li, P. B. Stetson et al., "Cepheid calibrations from the hubble space telescope of the luminosity of two recent type Ia supernovae and a redetermination of the hubble constant," Astrophysical Journal, vol. 627, no. 2, pp. 579-607, 2005.

[13] S. Webb, Measuring the Universe: The Cosmological Distance Ladder, Springer-Praxis, Chichester, UK, 1999.

[14] J. A. Peacock, Cosmological Physics, Cambridge University Press, Cambridge, UK, 1999.

[15] S. Weinberg, Gravitation and Cosmology: Principles and Applications of the General Theory of Relativity, Wiley, New York, NY, USA, 1972.

[16] S. Weinberg, Cosmology, Oxford University Press, Oxford, UK, 2008.

[17] B. Leibundgut, R. Schommer, M. Phillips et al., "Time dilation in the light curve of the distant type Ia supernova SN 1995K," Astrophysical Journal, vol. 466, no. 1, pp. L21-L24, 1996.

[18] A. G. Riess, A. V. Filippenko, D. C. Leonard et al., "Time dilation from spectral feature age measurements of type Ia supernovae," Astronomical Journal, vol. 114, no. 2, pp. 722729, 1997.

[19] R. J. Foley, A. V. Filippenko, D. C. Leonard, A. G. Riess, P. Nugent, and S. Perlmutter, "A definitive measurement of time dilation in the spectral evolution of the moderate-redshift type la supernova 1997ex," Astrophysical Journal, vol. 626, no. 1, pp. L11-L14, 2005.

[20] S. Blondin, T. M. Davis, K. Krisciunas et al., "Time dilation in type Ia supernova spectra at high redshift," Astrophysical Journal, vol. 682, no. 2, pp. 724-736, 2008.

[21] J. D. Anderson, P. A. Laing, E. L. Lau, A. S. Liu, M. M. Nieto, and S. G. Turyshev, "Indication, from pioneer 10/11, Galileo, and Ulysses data, of an apparent anomalous, weak, long-range acceleration," Physical Review Letters, vol. 81, no. 14, pp. 28582861, 1998.

[22] J. D. Anderson, E. L. Lau, S. G. Turyshev, P. A. Laing, and M. M. Nieto, "Search for a standard explanation of the pioneer anomaly," Modern Physics Letters A, vol. 17, no. 14, pp. 875$885,2002$.

[23] J. D. Anderson, P. A. Laing, E. L. Lau, A. S. Liu, M. M. Nieto, and S. G. Turyshev, "Study of the anomalous acceleration of Pioneer 10 and 11," Physical Review D, vol. 65, no. 8, Article ID 082004, 2002.

[24] S. G. Turyshev, M. M. Nieto, and J. D. Anderson, "Study of the Pioneer anomaly: a problem set," American Journal of Physics, vol. 73, no. 11, pp. 1033-1044, 2005.

[25] S. G. Turyshev, V. T. Toth, L. R. Kellogg, E. L. Lau, and K. J. Lee, "A study of the pioneer anomaly: new data and objectives for new investigation," International Journal of Modern Physics $D$, vol. 15 , no. 1 , pp. $1-55,2006$.

[26] V. T. Toth and S. G. Turyshev, "The Pioneer anomaly: seeking an explanation in newly recovered data," Canadian Journal of Physics, vol. 84, no. 12, pp. 1063-1087, 2006.

[27] V. T. Toth and S. G. Turyshev, "Pioneer anomaly: evaluating newly recovered data," in Proceedings of the 3rd Mexican Meeting on Mathematical and Experimental Physics, vol. 977, pp. 264-283, September 2007.

[28] S. G. Turyshev and V. T. Toth, "Physics engineering in the study of the pioneer anomaly," http://arxiv.org/abs/0710.0191.

[29] M. M. Nieto and J. D. Anderson, "Using early data to illuminate the Pioneer anomaly," Classical and Quantum Gravity, vol. 22, no. 24, pp. 5343-5354, 2005.

[30] M. M. Nieto and J. D. Anderson, "Search for a solution of the Pioneer anomaly," Contemporary Physics, vol. 48, no. 1, pp. 4154, 2007.

[31] S. G. Turyshev and V. T. Toth, "The pioneer anomaly," Living Reviews in Relativity, vol. 13, p. 4, 2010.

[32] S. G. Turyshev and V. T. Toth, "The pioneer anomaly in the light of new data," Space Science Reviews, vol. 148, no. 1-4, pp. 149-167, 2009.

[33] J. D. Anderson, S. G. Turyshev, and M. M. Nieto, "A mission to test the Pioneer anomaly," International Journal of Modern Physics D, vol. 11, no. 10, pp. 1545-1551, 2002.

[34] M. M. Nieto, S. G. Turyshev, and J. D. Anderson, "The pioneer anomaly: the data, its meaning, and a future test," in Proceedings of the 2nd Mexican Meeting on Mathematical and Experimental Physics, pp. 113-128, September 2004.

[35] H. Dittus, S. G. Turyshev, C. Lämmerzahl et al., "A mission to explore the Pioneer anomaly," in Proceedings of the European Space Agency, no. 588, pp. 3-10, 2005.

[36] M. M. Nieto and S. G. Turyshev, "Finding the origin of the pioneer anomaly," Classical and Quantum Gravity, vol. 21, no. 17, pp. 4005-4023, 2004.

[37] G. U. Varieschi, "Conformal cosmology and the pioneer anomaly," http://arxiv.org/abs/1010.3262.

[38] R. D. Reasenberg, I. I. Shapiro, P. E. MacNeil et al., "Viking relativity experiment-Verification of signal retardation by solar gravity," The Astrophysical Journal, vol. 234, p. L219, 1979. 
[39] I. I. Shapiro, R. D. Reasenberg, P. E. MacNeil et al., “The Viking relativity experiment," Journal of Geophysical Research., vol. 82, p. 4329, 1977.

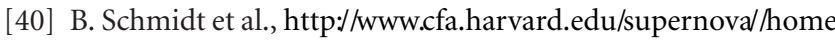
.html.

[41] S. Perlmutter et al., http://www.supernova.lbl.gov/.

[42] A. V. Filippenko, "Optical spectra of supernovae," Annual Review of Astronomy and Astrophysics, vol. 35, no. 1, pp. 309355, 1997.

[43] B. Leibundgut, "Cosmological implications from observations of type Ia supernovae," Annual Review of Astronomy and Astrophysics, vol. 39, no. 1, pp. 67-98, 2001.

[44] S. Perlmutter and B. P. Schmidt, Measuring cosmology with supernovae, Lecture Notes in Physics, Springer, Berlin, Germany, 2003.

[45] S. Perlmutter, G. Aldering, G. Goldhaber et al., "Measurements of $\Omega$ and $\Lambda$ from 42 high-redshift Supernovae," Astrophysical Journal, vol. 517, no. 2, pp. 565-586, 1999.

[46] A. G. Riess, A. V. Filippenko, P. Challis et al., "Observational evidence from supernovae for an accelerating universe and a cosmological constant," Astronomical Journal, vol. 116, no. 3, pp. 1009-1038, 1998.

[47] A. G. Riess, L. G. Sirolger, J. Tonry et al., "Type Ia supernova discoveries at $z \geq 1$ from the hubble space telescope: evidence for past deceleration and constraints on dark energy evolution," Astrophysical Journal, vol. 607, no. 2, pp. 665-687, 2004.

[48] A. G. Riess, L. G. Strolger, S. Casertano et al., "New hubble space telescope discoveries of Type La supernovae at $z \geq 1$ : narrowing constraints on the early behavior of dark energy," Astrophysical Journal, vol. 659, no. 1 I, pp. 98-121, 2007.

[49] http://braeburn.pha.jhu.edu/ ariess/R06/.

[50] S. M. Carroll, W. H. Press, and E. L. Turner, "The cosmological constant," Annual Review of Astronomy and Astrophysics, vol. 30, no. 1, pp. 499-542, 1992.

[51] A. G. Riess, W. H. Press, and R. P. Kirshner, "Using type Ia supernova light curve shapes to measure the Hubble constant," Astrophysical Journal, vol. 438, no. 1, pp. L17-L20, 1995.

[52] A. G. Riess, W. H. Press, and R. P. Kirshner, "A precise distance indicator: type la supernova multicolor light-curve shapes," Astrophysical Journal, vol. 473, no. 1, pp. 88-109, 1996.

[53] M. M. Phillips, "The absolute magnitudes of type Ia supernovae," Astrophysical Journal, vol. 413, no. 2, pp. L105-L108, 1993.

[54] L. M. Lubin and A. Sandage, "The Tolman surface brightness test for the reality of the expansion. IV. A measurement of the Tolman signal and the luminosity evolution of early-type galaxies," Astronomical Journal, vol. 122, no. 3, pp. 1084-1103, 2001.

[55] J. D. Barrow, The Constants of Nature, Pantheon Books, New York, NY, USA, 2002.

[56] L. B. Okun, "Fundamental units: physics and metrology," Lecture Notes in Physics, vol. 648, p. 57, 2004.

[57] J.-P. Uzan, "The fundamental constants and their variation: observational and theoretical status," Reviews of Modern Physics, vol. 75, no. 2, pp. 403-455, 2003.

[58] M. T. Murphy, J. K. Webb, V. V. Flambaum, C. W. Churchill, and J. X. Prochaska, "Possible evidence for a variable finestructure constant from QSO absorption lines: systematic errors," Monthly Notices of the Royal Astronomical Society, vol. 327, no. 4, pp. 1223-1236, 2001.

[59] A. Diaferio, L. Ostorero, and V. F. Cardone, "Gamma-ray bursts as cosmological probes: LambdaCDM vs. conformal gravity," http://arxiv.org/abs/1103.5501.
[60] M. N. U. Humason, M. L. Mayall, and A. R. Sandage, "Redshifts and magnitudes of extragalactic nebulae," The Astronomical Journal, vol. 61, pp. 97-162, 1956.

[61] J. B. Oke and A. Sandage, "Energy Distributions, K Corrections, and the Stebbins-Whitford Effect for Giant Elliptical Galaxies," The Astrophysical Journal, vol. 154, p. 21, 1968.

[62] A. Kim, A. Goobar, and S. Perlmutter, "A generalized K correction for type Ia supernovae: comparing R-band photometry beyond $\mathrm{z}=0.2$ with $\mathrm{B}, \mathrm{V}$, and R-band nearby photometry," Publications of the Astronomical Society of the Pacific, vol. 108, no. 720, pp. 190-201, 1996.

[63] A. Kim et al., "K Corrections For Type Ia Supernovae and a Test for Spatial Variation of the Hubble Constant," http://arxiv.org/abs/astro-ph/9602123.

[64] P. Nugent, A. Kim, and S. Perlmutter, "K-corrections and extinction corrections for type Ia supernovae," Publications of the Astronomical Society of the Pacific, vol. 114, no. 798, pp. 803-819, 2002. 

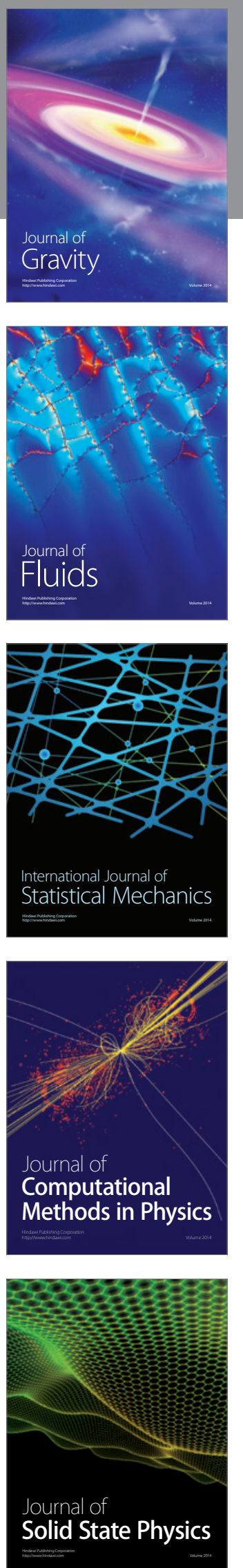

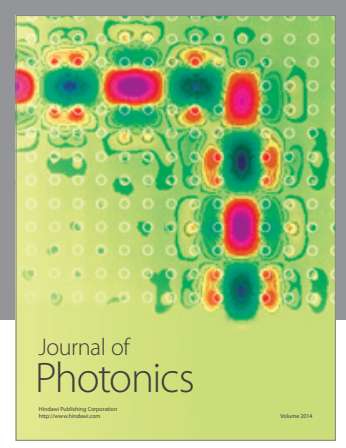

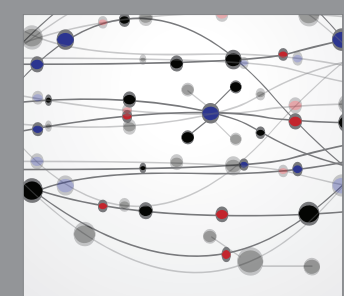

The Scientific World Journal
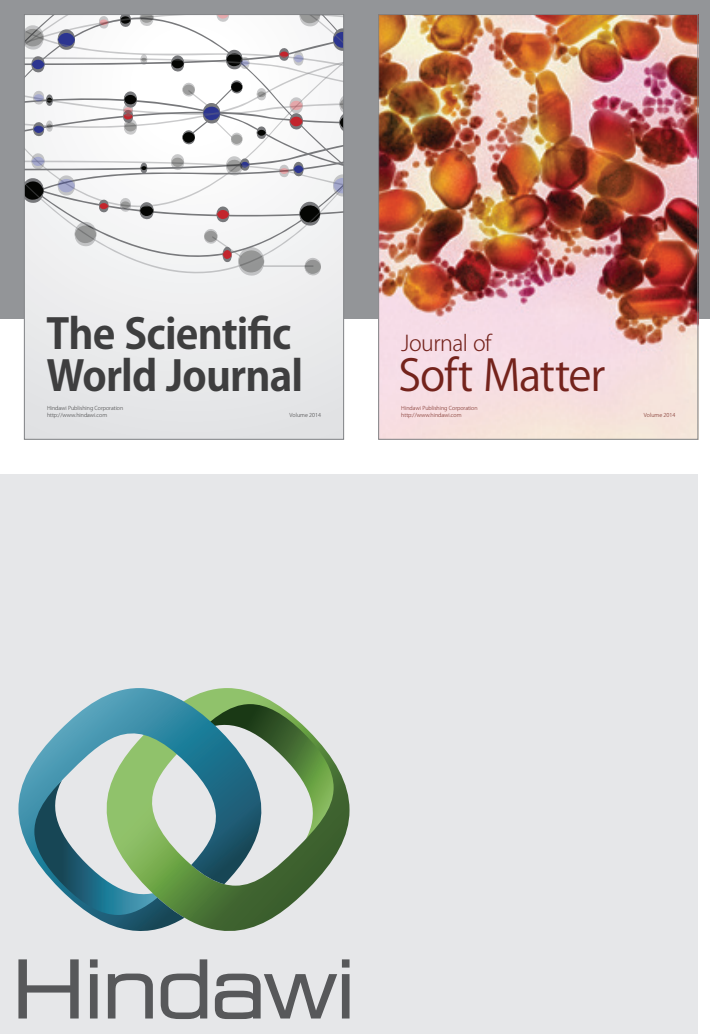

Submit your manuscripts at

http://www.hindawi.com
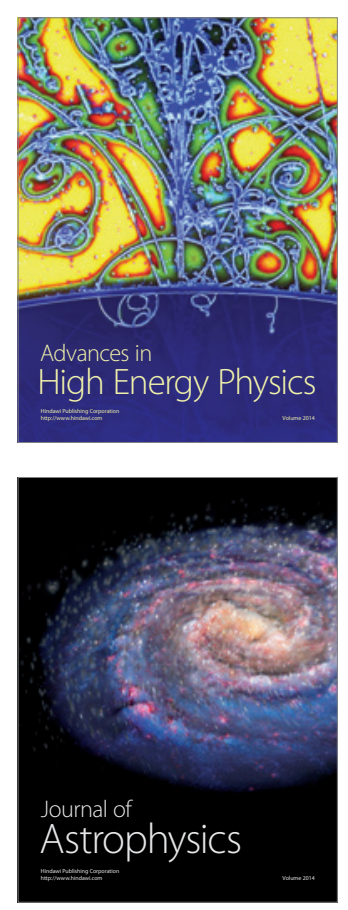
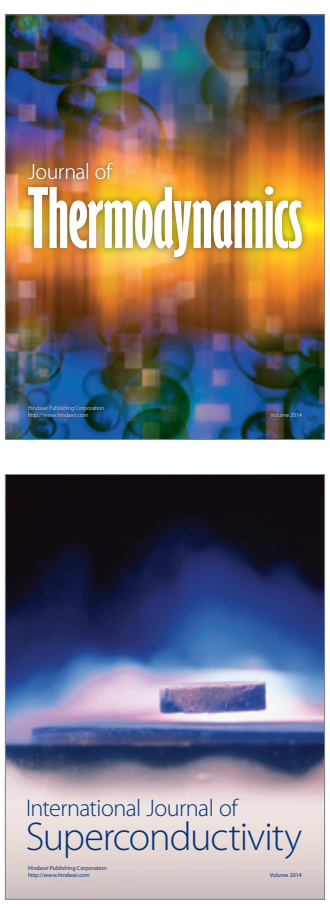
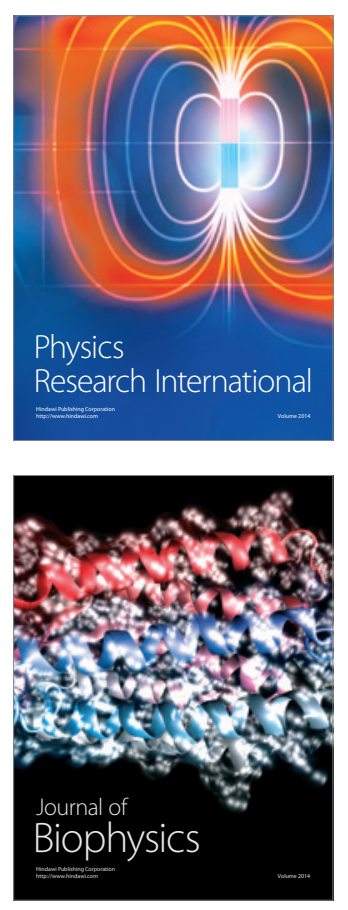
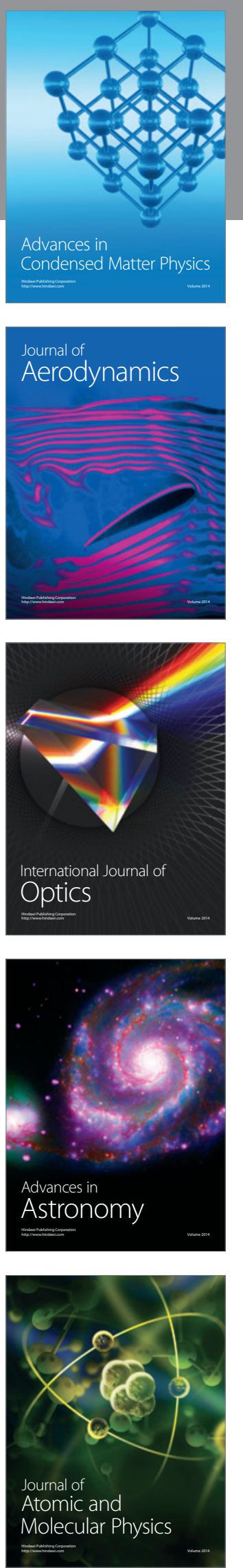